\title{
Spectroscopy Methods and Applications of the Tor Vergata Laser-Plasma Facility Driven by GW-Level Laser System
}

\author{
M. Francucci, P. Gaudio, S. Martellucci, and M. Richetta \\ Department of Mechanical Engineering, University of Rome "Tor Vergata”, Via del Politecnico 1, 00133 Rome, Italy \\ Correspondence should be addressed to P. Gaudio, gaudio@ing.uniroma2.it
}

Received 22 February 2011; Revised 15 April 2011; Accepted 3 May 2011

Academic Editor: Jozef Kaiser

Copyright () 2011 M. Francucci et al. This is an open access article distributed under the Creative Commons Attribution License, which permits unrestricted use, distribution, and reproduction in any medium, provided the original work is properly cited.

\begin{abstract}
A review focused on plasma induced on solid target by GW-level pulsed laser source is presented. A description of the Tor Vergata laser-plasma source (TVLPS), at the Tor Vergata University in Rome, is given. Such a facility uses a 1 GW, tabletop, multistage

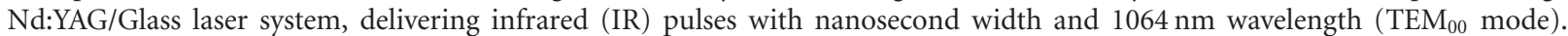
Its applications are discussed providing: wide analysis of IR $\rightarrow$ soft X-ray conversion efficiency $(1.3-1.55 \mathrm{keV})$; measures and modeling of line emission in soft X-ray spectra, such as those from zinc plasma near Ne-like Zn XXI and from barium plasma near Ni-like Ba XXIX. Particular attention is devoted to high- $n$ dielectronic Rydberg satellites for finding a useful diagnostic tool for plasma conditions. Dependence of plasma spectra on laser parameters is shown. Finally, microradiography applications are presented for thin biological samples. Images permit to visualize specific structures and detect bioaccumulation sites due to contamination from pollutants.
\end{abstract}

\section{Introduction}

Plasma [1] is a particular state of the matter beside ordinary solid, liquid, and gaseous ones usually called fourth state. It is constituted by neutral atoms, negative (electrons) and positive (ions) charged particles subjected to the action of long-range electromagnetic fields, governing the motion, and able to produce electric and magnetic forces.

The charge separation can be properly generated by heating the matter to high temperatures (around $10^{4}{ }^{\circ} \mathrm{C}$ at least), giving a validation of the plasma definition in terms of high-temperature ionized gas. It represents an interesting, original, very intricate matter state whose study has required the development of specific scientific disciplines and advanced research fields.

The survey of the matter in the plasma state is important, because it constitutes the $99 \%$ of the universe matter. Specifically, the stars (e.g., the sun) are the more common and prevailing examples of natural plasmas. Here, temperatures can achieve the hundreds of millions of degrees, and the produced high energies cannot be explained in terms of chemical reactions but only by nuclear fusion processes induced by high temperatures.
It is also possible to generate plasma in artificial way for scientific and industrial purposes. This usually occurs in research laboratories and industries, especially for surveys in the fields of the radiation-matter interaction, mechanical processing (welding, cutting, drilling, etc.), and material treatment, such as the plasma-enhanced chemical vapour deposition (PECVD). It is used to deposit thin films from a gas state (vapour) to a solid state onto a wafer often containing metal layers or other temperature-sensitive structures for applications in semiconductor manufacturing.

The production of artificial plasmas is relatively easy from a theoretical point of view, while it presents some difficulties for the practical realization due to the high energy that one has to supply to matter for producing its heating at high temperatures.

The more common techniques used for the artificial plasma generation are the following: electric discharge in a low-pressure gas and heavy heating of a solid, liquid, or gaseous target by means of a high-power focused laser beam.

Specifically, the application of the latter method has been more and more feasible thanks to the advent and the development in many basic research laboratories of highintensity, tabletop, pulsed laser systems [2-5] with unique 
properties that have opened new and unexpected frontiers in the plasma research field.

These experimental apparatuses are characterized by dimensions comparable to those of an optical table (whence the name "tabletop") and are based on an ultraintense pulsed (up to fs-level) laser source emitting high-power (up to PW-level) radiation usually in the infrared (IR), visible or ultraviolet (UV) region of the electromagnetic spectrum.

So, high-temperature plasma is generated when the laser pulse is focused on a target and the laser intensity reaches or exceeds the threshold for plasma generation (about $10^{7} \mathrm{~W} / \mathrm{cm}^{2}$ ) [4]).

Within this framework, laser-matter interaction for plasma generation is currently a topic of large and growing interest [6] that attracts various scientific research fields, such as plasma physics, laser physics, spectroscopy, and multiphoton physics, microlithography, and so forth.

More precisely, in the field of laser-induced plasmas, the main applications $[7,8]$ concern the traditional area of inertial confinement fusion $[9,10]$, astrophysics $[11,12]$, $\mathrm{X}$-ray spectroscopy $[4,13-20], \mathrm{X}$-ray imaging $[4,21-23]$, quantum electrodynamics and high-energy physics [24-26], atomic physics [27], solid-state physics [28], conversion efficiency studies from laser radiation to UV or X-rays $[4,5]$, evaluation of the energy of the radiation backscattered by plasma and of its reflectivity [4], and so on.

In this paper the attention will focus on a specific laserplasma source (TVLPS), installed and actually operating at the Quantum Electronics and Plasma Laboratory of the Tor Vergata University (Rome, Italy), based on a high-intensity, GW-level, multistage, pulsed, tabletop Nd:YAG/Glass laser. Specifically, in this paper some results and applications of this laser-plasma source are given concerning soft X-ray spectroscopy [18-20] and imaging, especially in the field of the microradiographies of thin biological samples [23].

Spatially resolved X-ray spectra from laser-induced plasma have been detected by using focusing spherically bent crystal spectrometers $[20,22]$ with spatial resolution of the order of tens of $\mu \mathrm{m}$ in the direction perpendicular to laser-plasma expansion and with a theoretical spectral resolving power $\lambda / \Delta \lambda$ of the order of some thousands up to a maximum of 10000 .

Within this framework, X-ray spectroscopy of multicharged ions plays a remarkable role, exploited at the TVLPS for the survey of high-temperature and high-density (close to that of a solid) matter.

For example, X-ray emission spectra both of He-like and Ne-like ions have been used as diagnostic tool for several types of plasmas, such as those of astrophysical interest [19]. Significant information in the field of plasma physics have been also obtained by means of model calculations and measurements of the X-ray emission spectra of a barium (Ba) plasma in spectral range of high- $n$ (principal quantum number) Rydberg levels in a near Ni-like Ba XXIX ionization state [20] and by the analysis of high- $n$ dielectronic Rydberg satellites in the near Ne-like Zn XXI ion spectra from Mg-like Zn XIX and Na-like Zn XX ions [18] (Section 4). A further observation emerges from the analysis of X-ray spectra (e.g., those due to near Ne-like Fe XVII ion stages), namely,

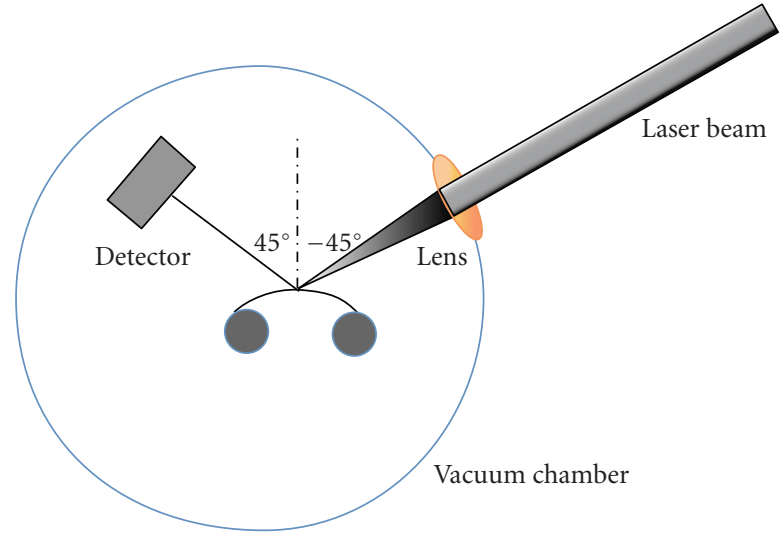

FIGURE 1: Schematic diagram of the experimental set up of the TVLPS.

a dependence of line strengths from laser intensity on the target affecting the plasma conditions (i.e., temperature).

The digital X-ray images of thin biological samples have been instead recorded by means of an astronomical grade, front-illuminated, high-sensitivity, high-resolution, nonstandard, home-assembled CCD camera [23], optimized for very low flux imaging and spectroscopy in the visible and $\mathrm{X}$-ray regions of the electromagnetic spectrum. It employs a $2 k \times 2 k$ device ( $15 \mu \mathrm{m}$ pixel-size) that can be cooled up to a temperature of $-80^{\circ} \mathrm{C}$ for reducing dark current.

Finally, conversion efficiency studies $[4,5]$ from infrared laser radiation to $\mathrm{X}$-rays emitted by laser induced plasma for different solid targets in the region (1.3-1.55) $\mathrm{keV}$ are also reported. These investigations are interesting for the energetic balance and performance parameters of the laserplasma source.

The paper is organized as follows. The description of the Tor Vergata laser-plasma setup will be reported in Section 2. The latter will be divided in three subsections concerning the Nd:YAG/Glass laser (Section 2.1) and the detection systems of the plasma emitted radiation (spherically bent crystal spectrometer-Section 2.2-and CCD camera-Section 2.3). Then, the studies of the IR $\rightarrow \mathrm{X}$ ray conversion efficiency are reported in Section 3, followed by the results of X-ray spectroscopy (Section 4) and X-ray imaging of thin biological samples for the detection of bioaccumulation sites due to contamination from pollutants such as heavy metals (Section 5). Finally, Section 6 is devoted to conclusions followed by The Acknowledgments section.

\section{Tor Vergata Laser-Plasma Source}

In this section, divided in three subsections, an exhaustive description of the TVLPS setup $[4,5,23]$, schematically showed in Figure 1, will be provided. It is used for the generation and analysis of radiation from laser induced plasma in the regions of visible, UV and X-rays.

Its main elements are a GW-level, multistage, pulsed, solid-state, infrared, tabletop Nd:YAG/Glass laser source, whose main characteristics will be reported in the next section, a double-stage vacuum chamber where plasma is 
generated, a vacuum pump system linked to the chamber, an ADC acquisition system (LeCroy 9361 Oscilloscope) for visualization and measurement of the characteristics of laser and plasma emitted radiation pulses, a fast photodiode for monitoring the temporal profile and the stability of laser pulse, and a series of constituents inside the vacuum chamber: a rotating tape target on whose surface a plasma is generated, a doublet/triplet lens for focusing the laser beam onto target surface, a mechanical support and a motor for target rotating, a spherically bent crystal spectrometerillustrated in the Section 2.2-for radiation spectral analysis, a nonstandard, home-assembled CCD camera-described in Section 2.3-for recording both plasma emission spectra and sample images, especially in the soft X-ray region and, finally, a commercial Quantrad PIN photodiode (model 100PIN-125N, bias voltage $=200 \mathrm{~V}$ ) for the detection of plasma radiation (mainly $\mathrm{UV}$ and $\mathrm{X}$ ).

In the apparatus, the rotating tape target is oriented in such a way to reduce the amount of ejected debris. More exactly, the normal to the target plane makes an angle of $-45^{\circ}$ compared to the direction of the incoming beam (i.e., the axis of the focusing lens) and $+45^{\circ}$ with respect to the detection system (Figure 1). The lens, used for focusing the laser beam on a target point inside the vacuum chamber which can operate at a very low pressure $\left(\leq 10^{-4} \mathrm{mbar}\right)$, is characterized by a focal length of about $f=20 \mathrm{~cm}$ and a diameter $d=5 \mathrm{~cm}$. So, the laser spot size achieves a limit value of about $100 \mu \mathrm{m}$ on the focal plane and the maximum pulse intensity $I$ is close to $10^{13} \mathrm{~W} / \mathrm{cm}^{2}$ in the best focus and highest (10 J) laser energy conditions.

This high-intensity laser light is firstly absorbed in a thin layer of the irradiated sample with a thickness of the order of skin depth. As a consequence, a heavy heating is observed in the target region where the laser beam is focused, followed by melting and vaporization. Then, a high-temperature artificial plasma is generated at target surface that tends to cool by expanding in the surrounding environment.

A gradient of the plasma density develops concurrently and laser energy can be absorbed by plasma for long-width laser pulses (higher than $10 \mathrm{ps}$ [4]). In these conditions, especially for long pulse length $\tau$ of the order of ns ( $15 \mathrm{~ns}$ for the TVLPS), an opacity phenomenon due to plasma self-absorption is also observed that strongly affects the characteristics of the plasma emission spectra, such as intensity and width of spectral lines [29].

Moreover, a so-produced hot and dense plasma behaves as a point-like and nonmonochromatic radiation source; that is, plasma emitted radiation is observed in a wide spectral range (visible, UV and X-rays) due to line transitions, radiative recombination and bremsstrahlung radiation $[30,31]$.

This radiation, opportunely detected by means of spectrometers, CCD cameras, and so forth, can be used for numerous applications and studies in the field of the lasermatter interaction with particular attention to the region of UV and X-rays, as specifically occurs for the TVLPS, where has been observed emission of radiation up to an energy of $5 \mathrm{keV}$ (soft X-rays) [23].

More precisely, radiative properties of hot and dense matter represent a noninterfering, powerful, diagnostic probe for investigating the plasma state. In fact, many fundamental information concerning this matter state (e.g., density, temperature, chemical composition, ionization balance, opacity, hot electron fraction, etc.) can be directly inferred by plasma emission spectrum, thus giving a valid explanation to the growing interest addressed to the development of laserplasma sources.

2.1. Nd:YAG/Glass Laser Source. The Nd:YAG/Glass laser system $[4,5]$, working at the Quantum Electronics and Plasma Laboratory, is showed in Figures 2 and 3. It is the basic element of the TVLPS and belongs to the category of the tabletop, GW-level, multistage, pulsed, solid-state laser operating in MOPA (Master Oscillator Power Amplifier) configuration.

An exhaustive characterization of laser beam in terms of quality factor $M^{2}$ and hot spots has been previously presented $[32,33]$. These studies have mainly shown that the quality of the laser beam deteriorates, passing from one amplification stage to the next one (see $M^{2}$ values in [32]). The situation is even worst when lamp voltage supply is increased, especially for the second amplifier. Finally, hot spots have been found along amplification chain and discussed in [33].

The laser source is "noncommercial" and its dimensions are comparable to those of an optical table, whence the name "tabletop". It delivers pulses in the NIR (near IR) with a wavelength $\lambda=1064 \mathrm{~nm}$ and a width $\tau=15 \mathrm{~ns}$ according to $\mathrm{TEM}_{00}$ emission mode and linear polarization P.

The laser facility is composed by a Nd:YAG oscillator, based on optical modulation by Pockels cell (Q-switched technique) and able to deliver light pulses with a maximum energy of $36 \mathrm{~mJ}$, followed by four amplification stageswhence the name "multistage" - that raise the pulse energy up to the highest value of $10 \mathrm{~J}$, corresponding to peak power close to $1 \mathrm{GW}$.

The first two amplifiers are also Nd:YAG, while the last ones are Nd:Glass, whence the name Nd:YAG/Glass given to the laser source. The dimensions of the amplifier rods are growing from the first to the latter amplification stage because of the natural and artificial-induced by proper beam expanders-laser beam spread, ultimately reaching a $2.5 \mathrm{~cm}$ maximum rod diameter in correspondence of the fourth amplifier.

The rod sizes are comparable to the laser spot propagating along the amplification chain, so enabling one to practically exploit all the active volume of the amplifiers for increasing the laser pulse energy.

The first amplifier is equipped-as the oscillator-with two flash lamps for the pumping of Nd:YAG crystal and can operate with a maximum pulse repetition rate (PRR) of $10 \mathrm{~Hz}$. It is also able to raise the laser energy up to a maximum value of $220 \mathrm{~mJ}$, corresponding to an energy gain of 9.8 in the optimal alignment conditions and for an input energy of $22.4 \mathrm{~mJ}$. This last value is that measured by taking into account the damping of laser beam coming from oscillator due to interaction with several optical elements (mirrors, beam expander, polarization plates, and Faraday rotator).

Similarly, the second amplifier (Nd:YAG) is equipped with four flash lamps and it is characterized by a maximum 


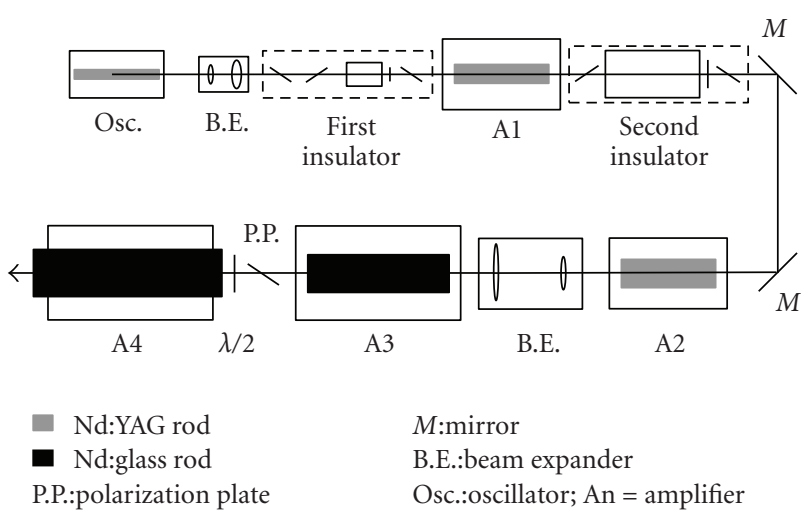

FIgURE 2: Scheme of our GW-level, tabletop, multistage, pulsed Nd:YAG/Glass laser source.

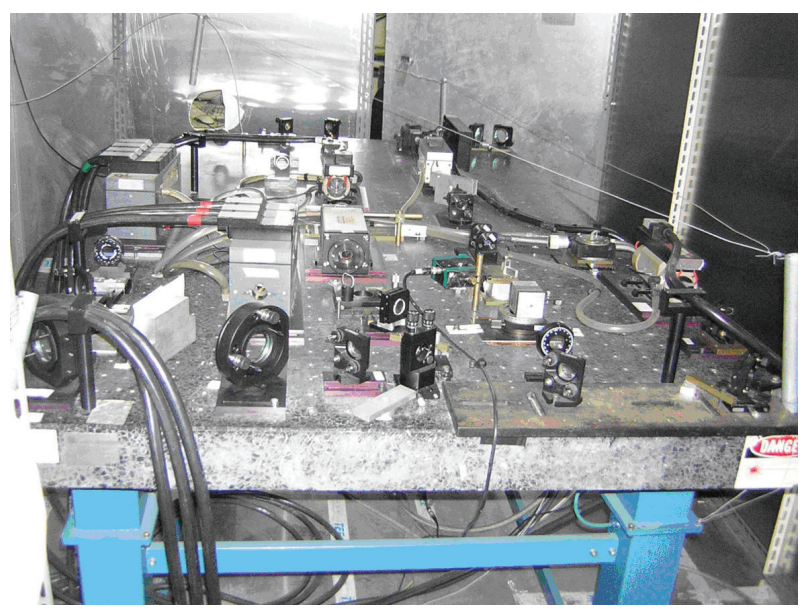

FIgURE 3: Photo of our GW-level pulse, tabletop, multistage Nd:YAG/Glass laser facility.

PRR of $10 \mathrm{~Hz}$ and a maximum gain of 4.4. So, it permits to increase the laser pulse energy up to $781 \mathrm{~mJ}$.

The third amplifier, instead, is equipped with a Nd:Glass crystal pumped by six flash lamps and can operate with a maximum PRR of 1 shot/minute though the optimal working PRR is fixed at 1 shot/4 minutes in order to minimize the thermal lens effects inside the crystal. This amplification stage increases the laser energy up to the value of $3.94 \mathrm{~J}$, corresponding to a maximum gain of 6.7.

Finally, the fourth amplifier (Nd:Glass) is equipped with eight flash lamps and it is characterized by a maximum PRR of $1 \mathrm{shot} / \mathrm{min}$, an optimal working PRR of 1 shot $/ 4$ minutes (such as the third amplifier) and highest gain of 3.5, so raising the laser pulse energy up to $10 \mathrm{~J}$.

The laser beam is then focused onto target surface by means of a computer-optimized doublet/triplet lens achieving a maximum intensity around $10^{13} \mathrm{~W} / \mathrm{cm}^{2}$ on the focal plane, sufficient to generate a hot and dense plasma inside the vacuum chamber emitting visible, UV and, especially, soft X radiation.

In the Nd:YAG/Glass laser source are also present two optical isolators. The former is located at the output of the oscillator, while the second isolator is placed between the first

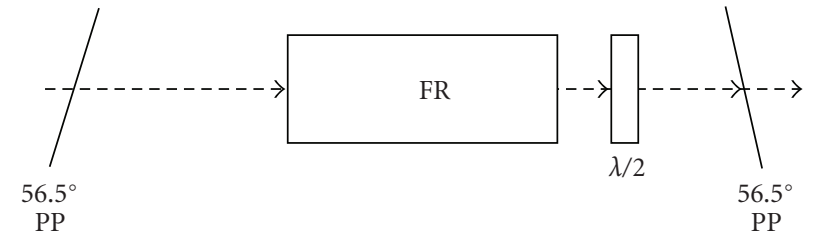

FIgure 4: Scheme of an optical isolator. Dashed line with arrows denotes the laser beam direction.

two amplifiers. Each of the two optical isolators is a system constituted by a Faraday rotator (FR) followed by $\lambda / 2$ and $56.5^{\circ}$ polarization plates (PPs), respectively, and by another $56.5^{\circ} \mathrm{PP}$ located before the Faraday rotator (Figure 4).

The optical isolators play a double and fundamental role for the optimization of laser performances: (1) rejection of the radiation retrodiffused by plasma, so avoiding the damaging of the optics; (2) reduction to a minimum of the interaction between the amplifiers in order to achieve conditions of optimal optical insulation, related to the rejection efficiency of the polarization plates.

In summary, resorting to a comparison with the electronics, such an insulation system behaves as an oneway switch that permits the passage (switch on) of the radiation with proper polarization state ( $\mathrm{P}$ in our case) travelling towards target, while stops (switch off) both the S-polarized light retrodiffused by target-characterized by a linear polarization state perpendicular to the plane of the optical table - and the radiation opportunely converted from $\mathrm{P}$ - to S-polarization state by means of the isolator.

The positions of these last ones along the amplification chain have been properly selected for strongly reducing the optical interaction of the amplifiers in the part of the laser source (oscillator and the first two amplifiers) where the energy gain is maximum and, thus, the effects of a worse optical insulation of the amplifiers are more evident.

It is clear that the ideal working condition is that realized by placing an optical isolator between every amplifier pair, though at the expense of a higher attenuation of laser energy.

Laser pulse, that is used as a trigger too, is monitored by a fast photodiode in order to measure the pulse energy and check the temporal stability. Besides this, for each laser shot the aforesaid LeCroy oscilloscope stores the plasma emitted radiation (mainly UV and X-rays) through a voltage signal showing the same temporal profile of the laser pulse with similar width (15 ns).

The plasma radiation is instead measured by the aforesaid Quantrad PIN photodiode placed inside the vacuum chamber. It forms a variable angle with respect to the target normal depending on working conditions and is characterized by a $0.3 \mu \mathrm{m}$ silicon input window, a $\mathrm{n}^{+}$doped cathode and an active intrinsic region of $125 \mu \mathrm{m}$.

In summary, we conclude this section by recalling the main features of our Nd:YAG/Glass laser system listed in the following Table 1.

2.2. Spherical Crystal-Based Spectrometer. The spectrometer $[20,22,34-36]$, used for the spectroscopy experiments in 
TABLE 1: Main specifications of the Tor Vergata Nd:YAG/Glass laser system.

\begin{tabular}{lc}
\hline \multicolumn{2}{c}{ Nd:YAG/Glass laser system specifications } \\
\hline Wavelength & $1064 \mathrm{~nm}$ \\
Pulse width & $15 \mathrm{~ns}$ \\
Maximum pulse energy & $10 \mathrm{~J}$ \\
Peak power & $1 \mathrm{GW}$ \\
Laser spot diameter on the focal plane about & $100 \mu \mathrm{m}$ \\
Maximum pulse intensity on the focal plane about & $10^{13} \mathrm{~W} / \mathrm{cm}^{2}$ \\
Emission mode & $\mathrm{TEM} 00$ \\
Linear polarization state & $\mathrm{P}$ \\
Optimal working PRR & 1 shot/4 minutes \\
\hline
\end{tabular}

the field of X-rays emitted by laser induced plasma, has been provided by Multicharged Ions Spectra Data Center of VNIIFTRI Institute (Moscow, Russia). It is characterized by the peculiar feature of being constituted by a mica or quartz spherical crystal, whence the name.

The device, particularly suitable for plasma microsources investigations, combines the focusing property of a spherical mirror with the Bragg diffraction of a crystal. A high spatial resolution, normally varying in the range $20-50 \mu \mathrm{m}$, along the direction perpendicular to light source (i.e., to plasma expansion in our case) represents its main characteristic along with a high spectral resolving power $\lambda / \Delta \lambda=$ 3000-10000.

The working principle is similar to that of a cylindrical crystal based Johan spectrometer with the advantage to produce a focusing of the incoming light on a plane perpendicular to the spectral one. So, a 1D (one-dimensional) image of the light source along the direction perpendicular to the spectrum one can be obtained.

In order to better understand the working principle, let us consider a spherical crystal of radius $R_{c}$ and the following Bragg law stating the X-ray diffraction by the crystal

$$
2 d \sin \theta=n \lambda \text {. }
$$

Here, $d$ denotes the lattice spacing, $\lambda$ the wavelength of the incoming light (e.g., $\mathrm{X}$-rays), $\theta$ the Bragg angle- that is, the complementary angle of the incident one, $\alpha$, measured with respect to the normal to the crystal surface-and $n$ is the diffraction order $(n=1,2,3, \ldots)$.

Let us also draw several straight lines incoming on various points of the spherical crystal with the same Bragg angle. All these lines will cross in a same point $P$ that belongs to the so-called Rowland circle tangent to the crystal (Figure 5) and whose curvature radius $R$ takes the form

$$
R=\frac{R_{c}}{2} .
$$

So, by locating a polychromatic radiation source at the point $\mathrm{P}$, the emitted light rays hit the crystal with the same Bragg angle and only the wavelength corresponding to this angle is diffracted by the crystal (1).

In summary, for radiation coming from a generic point $\mathrm{P}$ of the Rowland circle, a one-to-one relationship exists

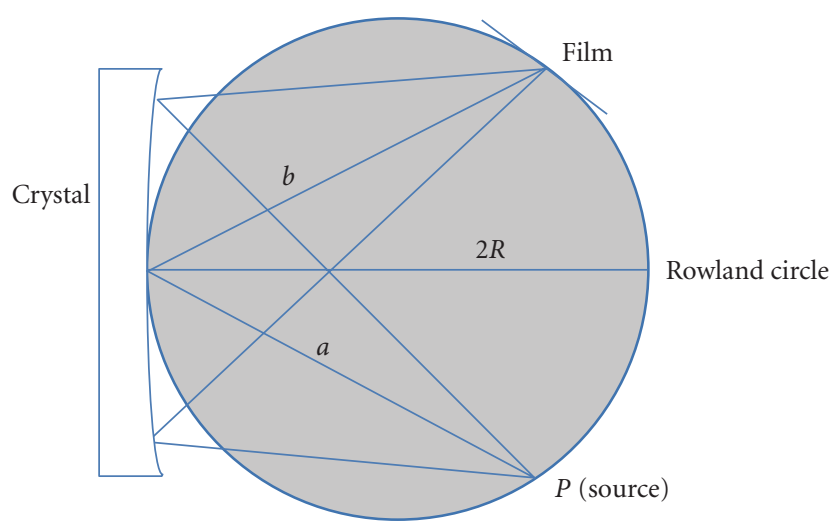

FIGURE 5: Scheme of the working principle of a spectrometer based on spherical crystal with relative Rowland circle.

between the circle points and the diffracted wavelength (Figure 5).

Thus, by placing a detector (film, CCD camera, etc.) tangent to the Rowland circle, only the rays reflected by the crystal with a specific wavelength hit the receiver for any position of light source along the Rowland circle. Nevertheless, since the crystal is spherical, the source location can be chosen in such way that, on the sagittal plane perpendicular to the Rowland circle, a 1D image of the source is sharpened on the detector with suitable magnification.

As a consequence, it is necessary to find the condition for obtaining such a 1D image by using a spherical crystal. This condition is stated in terms of the evaluation of the distance $a$ between the light source and the crystal.

Specifically, in order to achieve this result, the focusing mirror law is used. It reads

$$
\frac{1}{a}+\frac{1}{b}=\frac{1}{f}
$$

Here, $b$ is the crystal-detector spatial separation given by

$$
b=2 R \sin \theta,
$$

while $f$ is the focal length of the spherical crystal on the sagittal plane taking the form

$$
f=\frac{R}{\sin \theta} .
$$

By inverting (3) and taking into account (4) and (5), the following explicit formula for $a$ can be derived:

$$
a=\left|\frac{2 R \sin \theta}{\cos (2 \theta)}\right| .
$$

As a result, a 1D image of the light source is obtained by means of a spherically bent crystal spectrometer whose spectral dynamics depends on sizes, curvature radius, and lattice spacing of the crystal, beside the Bragg angle.

The spectral range is also easily found. In fact, it is sufficient to know where rays coming from the source cross the Rowland circle and, then, to recall the one-to-one 


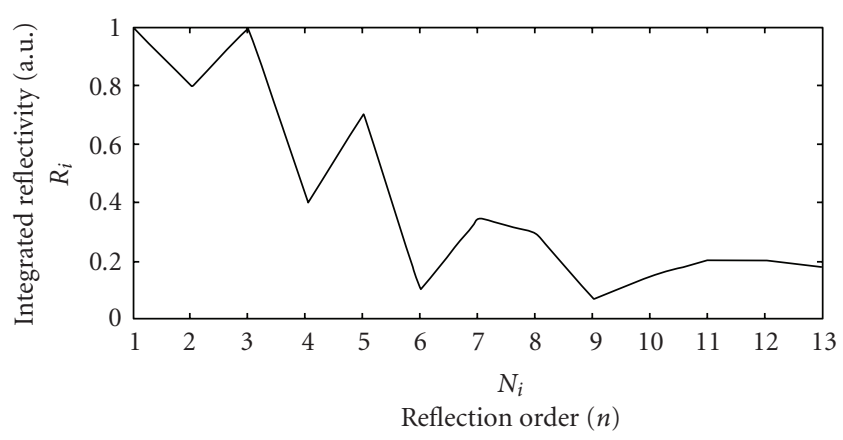

FIgURE 6: Mica reflectivity versus the diffraction order $n$.

relationship between circle points and diffracted wavelength, that is to evaluate the angle among these points and the surface of crystal (for instance at the center of the latter).

A mica spherical crystal is employed in our spectrometer. It is characterized by a lattice spacing $2 d=19.9149 \AA-$ using the 002 lattice plane-corresponding to an energy of $622 \mathrm{eV}$. Nonetheless, in order to avoid that the detector (RAR film, CCD, etc.) intercepts radiation incoming on the crystal, it is necessary to work with an incidence angle $\alpha$ of $10^{\circ}$ at least, that is, with an minimum energy of $622 \mathrm{eV} / \cos \left(10^{\circ}\right)$ $\cong 632 \mathrm{eV}$.

Furthermore, the wavelength $\lambda$ incident on the spherical crystal can be continuously changed within a factor equal to 2 by varying the $\theta$ Bragg angle. Actually, it is possible to attain energies up to $10 \mathrm{keV}$ (1) by means of the particular feature of the mica of having a high reflectivity also for high diffraction order (Figure 6). So, the energy range where a mica spherical crystal can be used is up to $10 \mathrm{keV}$.

Such a property justifies the frequent employment of the mica for the realization of a spherically bent crystal with the purpose to make high spatial and spectral resolution X-ray spectroscopy in a wide spectral range.

Beside the main application in the field of the X-ray emission spectroscopy, other interesting employments of a spherical crystal spectrometer concern: (a) 1D imaging of the light source arranging the device according to (6) and (b) 2D (two-dimensional) imaging of the light source, locating both the latter and the detector outside of the Rowland circle according to (3).

However, it is still possible to obtain 1D images of the light source, though nonperfectly focused, by moving away slightly the detector from Rowland circle.

Moreover, a spherically bent crystal spectrometer is characterized by a higher brightness with respect to the traditional Johan spectrometers due to the crystal roundness. This property is very useful when one works with radiation damped because of long distances travelled in air.

Such an instrument can be also used as monochromatic mirror in order to focus X-rays emitted by a source (e.g., laser induced plasma) on a small area.

Another possible and interesting application concerns the use of two spherical crystal spectrometers for producing a parallel X-ray beam. In order to achieve this result, it is sufficient to place the focal point of the first crystal in correspondence of the focus of the second one.
TABLE 2: Main features of the spectrometer based on mica spherical crystal usually employed in our spectroscopy experiments. Note that the acronym FSSR means focusing spectrometer with $1 \mathrm{D}$ or 2D spatial resolution.

\begin{tabular}{|c|c|}
\hline \multicolumn{2}{|c|}{ Specifications of spherically bent mica crystal spectrometer } \\
\hline Spectrometer model & FSSR-JG-100 \\
\hline Crystal lattice spacing $(2 d)$ & $19.9149 \AA$ \\
\hline $\begin{array}{l}\text { Crystal curvature radius } \\
\left(R_{c}\right)\end{array}$ & $100 \mathrm{~mm}$ \\
\hline Crystal length $\left(L_{c}\right)$ & $26 \mathrm{~mm}(+2 \mathrm{~mm}$ in side the box $)$ \\
\hline Crystal width $\left(l_{c}\right)$ & $8 \mathrm{~mm}(+2 \mathrm{~mm}$ inside the box $)$ \\
\hline $\begin{array}{l}\text { Bragg angle at the center of } \\
\text { the crystal }\left(\theta_{0}\right)\end{array}$ & $50^{\circ}$ \\
\hline $\begin{array}{l}\text { Maximum spatial } \\
\text { resolution }(\Delta x)\end{array}$ & $\begin{array}{l}\text { (10-20) } \mu \mathrm{m} \text { (limited by the pixel or } \\
\text { film grain sizes) }\end{array}$ \\
\hline $\begin{array}{l}\text { Spectral resolution }(\Delta \lambda) \\
\text { about }\end{array}$ & $\begin{array}{l}4 \mathrm{~m} \AA \text {, that is } \Delta \lambda / \lambda \cong 3 \cdot 10^{-4} \Rightarrow \lambda / \Delta \lambda \cong \\
3333\end{array}$ \\
\hline Spectral range & up to $10 \mathrm{keV}$ \\
\hline
\end{tabular}

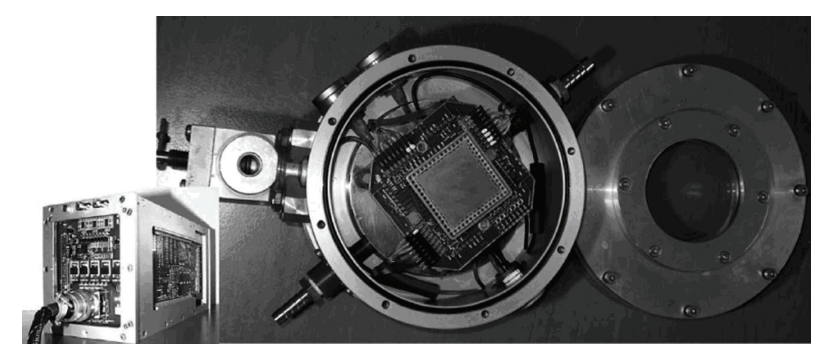

FIGURE 7: CCD camera with Loral laboratory chip and, on the left, SDSU controller.

In Table 2, a list of the main specifications of the mica spherical crystal based spectrometer, usually used in our spectroscopy experiments exploiting X-rays emitted by laser induced plasma, is reported.

2.3. CCD Camera. The device can be used in X-ray spectroscopy and imaging and is depicted in Figure 7, while its arrangement inside the vacuum chamber during the experiments is showed in Figure 8.

It is represented by an astronomical grade, front illuminated, high-sensitivity, high-resolution, nonstandard, homeassembled CCD camera [23] based on a Loral chip with a spatial resolution of $2 k \times 2 k$ pixels $(15 \mu \mathrm{m}$ pixel-size $)$ and a good cosmetic and photometric quality. The device also presents a satisfactory $\mathrm{X}$-ray response, equal to a maximum value around $70 \%$ in the spectral range (1-8) $\mathrm{keV}$ [23].

The instrument has been designed and tested at the Lick Observatory of the University of Santa Cruz (California, USA) for optimizing the detection of visible and $\mathrm{X}$ radiation with the purpose to develop astronomical focal devices.

The Loral chip, that can operate under the so-called inverted mode (multiphase-pinned mode, MPP [37]) at a temperature of $200 \mathrm{~K}$, shows a low dark current less than $0.1 \mathrm{e}-/ \mathrm{min}$, so permitting to obtain high-reliability and highaccuracy photometric digital images even when the CCD 


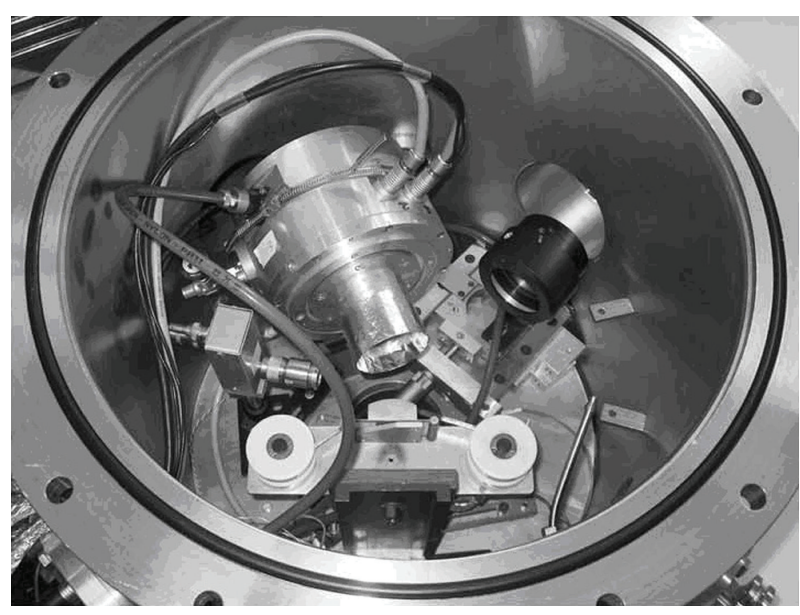

Figure 8: Arrangement of the Loral CCD camera, focusing lens (triplet), rotating tape solid target and Quantrad PIN photodiode inside the vacuum chamber adopted in the X-ray imaging experiments.

is not at low cryogenic temperatures where optimal working conditions should be achieved.

MPP represents a CCD working modality that we have adopted in our X-ray imaging experiments. Though MPP is responsible for a reduction of the full-well capacity, normally of the order of 120000 electrons, it presents many operative advantages such as

(a) remarkable reduction of dark current,

(b) optimal charge transfer efficiency (CTE),

(c) absence of surface residual image,

(d) excellent pixel-to-pixel uniformity,

(e) enhanced radiation hardness,

(f) very quickly CCD data erasure.

The CCD thermal dark current has been also decreased by means of a new, purposely-developed thermoelectric cooler system (TEC) which permits to reach operating cryogenic temperatures up to $-80^{\circ} \mathrm{C}$ under very low pressure conditions (about $10^{-6} \mathrm{mbar}$ ) within temperature fluctuations of $0.5^{\circ} \mathrm{C}$.

However, in our experiments, CCD camera has been cooled at higher temperatures (up to $-50^{\circ} \mathrm{C}$ ) than the optimal ones $\left(-80^{\circ} \mathrm{C}\right)$ because of a nonexcellent vacuum achieved in the camera corresponding to a pressure of $10^{-4} \mathrm{mbar}$ against $10^{-6} \mathrm{mbar}$ theoretically expected. Moreover, for the experimental tests, a CCD camera representing an upgrade of the systems $[38,39]$ has been actually used.

The electronics supporting CCD camera comprises the San Diego State University (SDSU) controller boards (Figure 7), that allow to handle a wide variety of imaging arrays from IR to X-rays, and some additional custom boards, while each operational parameter of the CCD is accessible through software.

In addition, the electronics drives CCD arrays-each containing one or more readout circuits_-in a fast readout mode up to $0.5 \mu$ s per pixel and permits to efficiently support many readouts, simultaneously.

A high dynamic range (16 bit) is also typical of these CCD cameras. It is obtained by the use of low-noise techniques and 16 bit A/D converters. The flexibility of the design also allows to control via computer a variable number of readouts. So, a flexible operation in time-resolved experiments is attained.

The CCD camera is controlled through a series of commands that are created by a VOODOO user application. The control and acquisition software for CCD system consists of two layers: the Java code layer and the C library layer. Finally, in order to process CCD images, IRAF and IDL software can be used.

In this framework, more details on SDSU and employed software can be found in [23].

\section{Results: IR $\rightarrow$ X-Ray Conversion Efficiency}

In this section, an estimation of the soft X-ray energy $E_{x}$ emitted by the TVLPS is reported in order to evaluate the conversion efficiency $\eta$ from IR laser radiation to soft Xphotons in the spectral region $(1.3-1.55) \mathrm{keV}$, that is, (89.56) $\AA$ in terms of wavelength.

The determination of X-ray energy emitted on the half solid angle from plasma source for each laser shot has been obtained by using the following formula $[4,5]$, assuming to calculate this energy at a distance $d_{s}=0.1 \mathrm{~mm}$ from expanding plasma source with an emission surface $A_{s}$ supposed reasonably corresponding to the circular area of laser spot on the target with radius around $100 \mu \mathrm{m}$ in the best focus conditions

$$
E_{x}=F_{x}\left(\frac{d}{d_{s}}\right)^{2} A_{s}[\mathrm{~J}]
$$

where

$$
F_{x} \cong 3.93 \frac{V_{x}}{R_{L}} \frac{\tau_{x}}{S_{\mathrm{xrd}}} \frac{1}{A_{\exp } T_{f}}\left[\mathrm{~J} / \mathrm{m}^{2}\right],
$$

is the X-ray fluence emitted by plasma source on the half solid angle falling onto Quantrad PIN photodiode at a distance $d$.

Here, $V_{x}$ denotes the amplitude of the signal due to Xphotons coming from laser generated plasma on various solid targets, read by means of oscilloscope, $A_{\text {exp }}$ the sensitive area of X-ray detector, $R_{L}$ the load where the X-ray detector is connected, $\tau_{x}$ the X-pulse FWHM (Full Width Half Maximum), $\mathrm{S}_{\mathrm{xrd}}$ the X-ray sensor responsivity $(\mathrm{C} / \mathrm{J}), \mathrm{T}_{f}$ the transmission coefficient of X-ray sensor and, finally, $d$ the plasma-detector distance along the normal-that is, where $\mathrm{X}$-ray emission is maximum-direction relative to target in the point where plasma is produced.

From (7) and (8) it is evident that $E_{x} \propto V_{x}$, that is, the $\mathrm{X}$-ray energy is directly proportional to the measured voltage signal.

In our experiments, one has $A_{\exp } \cong 100 \mathrm{~mm}^{2}, R_{L}=50 \Omega$, and $T_{f}=0.008$ in first approximation due to an $\mathrm{Al}$ filter with a thickness of $40 \mu \mathrm{m}$ placed in front of PIN photodiode (Figure 9), $S_{\text {xrd }} \cong 0.25 \mathrm{C} / \mathrm{J}$ evaluated by calibration curve of 


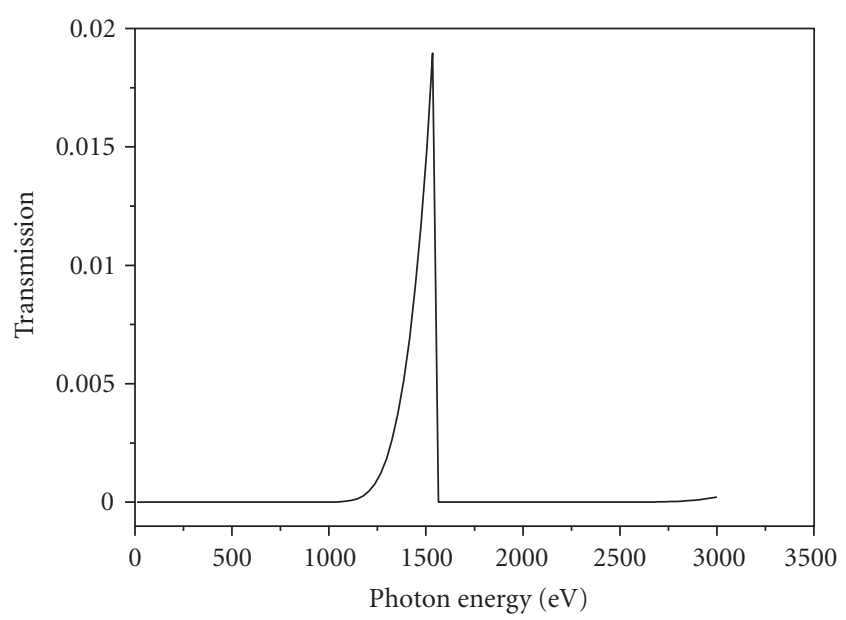

Figure 9: Transmission coefficient of a $40 \mu \mathrm{m}$ aluminum filter versus the energy of the incoming photons in the spectral region up to $3 \mathrm{keV}$.

the latter in the region around $1.5 \mathrm{keV}, \tau_{x} \cong 15 \mathrm{~ns}$ directly measured by oscilloscope and, finally, $d=0.35 \mathrm{~m}$.

Moreover, six different solid targets have been used for plasma generation and $\eta$ estimation: magnesium $(\mathrm{Mg}$, atomic number $Z=12$ ), titanium ( $\mathrm{Ti}, Z=22)$, iron (Fe, $Z=26)$, copper $(\mathrm{Cu}, Z=29)$, zinc $(Z n, Z=30)$, and yttrium $(\mathrm{Y}, \mathrm{Z}=39)$.

So, knowing the energy $E_{x}(7)$ of X-radiation emitted by laser-induced plasma, IR laser light $\rightarrow$ soft X-ray conversion efficiency can be easily calculated for each laser shot taking the form

$$
\eta=\frac{E_{x}}{E_{\mathrm{IR}}}
$$

Here, $E_{\mathrm{IR}}$ represents the laser energy illuminating the target experimentally measured, properly varying in the range (3.25-9.91) $\mathrm{J}$ and corresponding to a laser intensity $I$ on the focal plane between $2.76 \cdot 10^{12} \mathrm{~W} / \mathrm{cm}^{2}$ and $8.41 \cdot 10^{12} \mathrm{~W} / \mathrm{cm}^{2}$ with the focusing lens always in the best focus position (laser spot size on target of about $100 \mu \mathrm{m}$ ).

The values calculated for $\eta$ are a valid-yet slightly imprecise-estimation of the real quantities due to four different reasons: (1) exact evaluation of the distance between PIN photodiode and target point where plasma is generated, (2) position of PIN photodiode that not precisely corresponds to the target normal direction, so measuring a soft X-ray energy slightly less than that theoretically expected by means of (7), (3) exact evaluation of the position of the expanding plasma source, and (4) determination of correct value of the transmission coefficient in front of X-ray PIN diode.

In the tables that follow (i.e., Tables 3-8), the quantities $E_{\mathrm{IR}}$ (laser energy), $I$ (laser intensity), $V_{x}$ (amplitude of X-ray signal measured as average on four laser shots), $E_{x}$ (soft X-ray energy), $\eta$ (IR laser light $\rightarrow$ soft X-ray conversion efficiency), and $\eta_{\text {rel }}$ (relative conversion efficiency with respect to the target having the lowest value of $\eta$, i.e., yttrium in our case)
TABLE 3: Experimental measurements and results obtained for yttrium $(Z=39)$ by using the TVLPS.

\begin{tabular}{lcccc}
\hline$E_{\mathrm{IR}}(\mathrm{J})$ & $I\left(\mathrm{~W} / \mathrm{cm}^{2}\right)$ & $V_{x}(\mathrm{~V})$ & $E_{x}(\mathrm{~mJ})$ & $\eta_{Y}(\%)$ \\
\hline 3.2 & $2.76 \cdot 10^{12}$ & 0.41 & 0.22 & 0.0068 \\
4.5 & $3.83 \cdot 10^{12}$ & 1.1 & 0.59 & 0.013 \\
6.0 & $5.07 \cdot 10^{12}$ & 2.0 & 1.1 & 0.018 \\
7.4 & $6.32 \cdot 10^{12}$ & 2.8 & 1.5 & 0.020 \\
8.8 & $7.50 \cdot 10^{12}$ & 3.5 & 1.9 & 0.021 \\
9.9 & $8.41 \cdot 10^{12}$ & 4.2 & 2.3 & 0.023 \\
\hline
\end{tabular}

TABle 4: Experimental measurements and results obtained for titanium $(Z=22)$ by using the TVLPS.

\begin{tabular}{lccccc}
\hline$E_{\mathrm{IR}}(\mathrm{J})$ & $I\left(\mathrm{~W} / \mathrm{cm}^{2}\right)$ & $V_{x}(\mathrm{~V})$ & $E_{x}(\mathrm{~mJ})$ & $\eta_{\mathrm{Ti}}(\%)$ & $\eta_{\text {rel }}=\eta_{\mathrm{Ti}} / \eta_{\mathrm{Y}}$ \\
\hline 3.2 & $2.76 \cdot 10^{12}$ & 2.9 & 1.6 & 0.049 & 7.2 \\
4.5 & $3.83 \cdot 10^{12}$ & 5.1 & 2.8 & 0.062 & 4.8 \\
6.0 & $5.07 \cdot 10^{12}$ & 7.5 & 4.2 & 0.070 & 3.9 \\
7.4 & $6.32 \cdot 10^{12}$ & 11.0 & 5.9 & 0.079 & 4.0 \\
8.8 & $7.50 \cdot 10^{12}$ & 13.6 & 7.3 & 0.083 & 4.0 \\
9.9 & $8.41 \cdot 10^{12}$ & 15.4 & 8.3 & 0.084 & 3.7 \\
\hline
\end{tabular}

TABLE 5: Experimental measurements and results attained for iron $(Z=26)$ by using the TVLPS.

\begin{tabular}{lccccc}
\hline$E_{\mathrm{IR}}(\mathrm{J})$ & $I\left(\mathrm{~W} / \mathrm{cm}^{2}\right)$ & $V_{x}(\mathrm{~V})$ & $E_{x}(\mathrm{~mJ})$ & $\eta_{\mathrm{Fe}}(\%)$ & $\eta_{\mathrm{rel}}=\eta_{\mathrm{Fe}} / \eta_{\mathrm{Y}}$ \\
\hline 3.2 & $2.76 \cdot 10^{12}$ & 5.75 & 3.1 & 0.095 & 14.0 \\
4.5 & $3.83 \cdot 10^{12}$ & 8.94 & 4.8 & 0.11 & 8.5 \\
6.0 & $5.07 \cdot 10^{12}$ & 14.9 & 8.0 & 0.13 & 7.2 \\
7.4 & $6.32 \cdot 10^{12}$ & 19.9 & 10.7 & 0.14 & 7.0 \\
8.8 & $7.50 \cdot 10^{12}$ & 23.5 & 12.7 & 0.14 & 6.7 \\
9.9 & $8.41 \cdot 10^{12}$ & 27.9 & 15.0 & 0.15 & 6.5 \\
\hline
\end{tabular}

TABle 6: Experimental measurements and results attained for magnesium $(Z=12)$ by using the TVLPS.

\begin{tabular}{lccccc}
\hline$E_{\mathrm{IR}}(\mathrm{J})$ & $I\left(\mathrm{~W} / \mathrm{cm}^{2}\right)$ & $V_{x}(\mathrm{~V})$ & $E_{x}(\mathrm{~mJ})$ & $\eta_{\mathrm{Mg}}(\%)$ & $\eta_{\mathrm{rel}}=\eta_{\mathrm{Mg}} / \eta_{\mathrm{Y}}$ \\
\hline 3.2 & $2.76 \cdot 10^{12}$ & 5.0 & 2.7 & 0.083 & 12.2 \\
4.5 & $3.83 \cdot 10^{12}$ & 11.0 & 5.9 & 0.13 & 10.0 \\
6.0 & $5.07 \cdot 10^{12}$ & 17.9 & 9.6 & 0.16 & 8.9 \\
7.4 & $6.32 \cdot 10^{12}$ & 25.3 & 13.6 & 0.18 & 9.0 \\
8.8 & $7.50 \cdot 10^{12}$ & 33.5 & 18.1 & 0.20 & 9.5 \\
9.9 & $8.41 \cdot 10^{12}$ & 39.0 & 21.0 & 0.21 & 9.1 \\
\hline
\end{tabular}

are reported for the six solid targets used in the experiments. A maximum fluctuation of $5 \%$ has to be considered for the values of laser energy reported in the tables.

Finally, the atomic number $(Z)$ and the quantities $\left\langle V_{x}\right\rangle,\left\langle E_{x}\right\rangle$ and $\langle\eta\rangle$-representing the average of the values reported in the Tables 3-8-are shown in the last table (Table 9) for each considered element in order to evaluate the target with the best conversion efficiency in the analyzed spectral range.

The main results presented in the previous tables are shown from Figures 10 to 12 in order to better illustrate the 
TABLE 7: Experimental measurements and results attained for copper $(Z=29)$ by using the TVLPS.

\begin{tabular}{lccccc}
\hline$E_{\mathrm{IR}}(\mathrm{J})$ & $I\left(\mathrm{~W} / \mathrm{cm}^{2}\right)$ & $V_{x}(\mathrm{~V})$ & $E_{x}(\mathrm{~mJ})$ & $\eta_{\mathrm{Cu}}(\%)$ & $\eta_{\mathrm{rel}}=\eta_{\mathrm{Cu}} / \eta_{\mathrm{Y}}$ \\
\hline 3.2 & $2.76 \cdot 10^{12}$ & 11.6 & 6.2 & 0.19 & 28.0 \\
4.5 & $3.83 \cdot 10^{12}$ & 19.7 & 10.6 & 0.24 & 18.5 \\
6.0 & $5.07 \cdot 10^{12}$ & 31.6 & 17.0 & 0.28 & 15.6 \\
7.4 & $6.32 \cdot 10^{12}$ & 47.5 & 25.6 & 0.34 & 17.0 \\
8.8 & $7.50 \cdot 10^{12}$ & 58.6 & 31.6 & 0.36 & 17.1 \\
9.9 & $8.41 \cdot 10^{12}$ & 71.5 & 38.5 & 0.39 & 17.0 \\
\hline
\end{tabular}

TABLE 8: Experimental measurements and results obtained for zinc $(Z=30)$ by using the TVLPS.

\begin{tabular}{lccccc}
\hline$E_{\mathrm{IR}}(\mathrm{J})$ & $I\left(\mathrm{~W} / \mathrm{cm}^{2}\right)$ & $V_{x}(\mathrm{~V})$ & $E_{x}(\mathrm{~mJ})$ & $\eta_{\mathrm{Zn}}(\%)$ & $\eta_{\mathrm{rel}}=\eta_{\mathrm{Zn}} / \eta_{Y}$ \\
\hline 3.2 & $2.76 \cdot 10^{12}$ & 10.3 & 5.5 & 0.17 & 25.0 \\
4.5 & $3.83 \cdot 10^{12}$ & 21.3 & 11.5 & 0.25 & 19.2 \\
6.0 & $5.07 \cdot 10^{12}$ & 32.7 & 17.6 & 0.29 & 16.1 \\
7.4 & $6.32 \cdot 10^{12}$ & 49.1 & 26.5 & 0.36 & 18.0 \\
8.8 & $7.50 \cdot 10^{12}$ & 59.8 & 32.2 & 0.36 & 17.1 \\
9.9 & $8.41 \cdot 10^{12}$ & 70.0 & 37.7 & 0.38 & 16.5 \\
\hline
\end{tabular}

meaning of the study performed in the field of the IR laser light $\rightarrow$ soft X-ray conversion efficiency by using the Tor Vergata laser-plasma facility.

In summary, from tables and figures reported in this section along with literature information the followig is evident.

(a) The conversion efficiency $\eta$ from IR laser light to soft X-rays depends on the used target since it is related to the resonance line position-varying for different elements (e.g., lines 5C and 5D for $\mathrm{Cu}$ $[13,29]$ and lines $4 \mathrm{C}$ and $4 \mathrm{D}$ for $\mathrm{Zn}[18]$, as also shown in Figure 21, falling into the spectral range $1.3-1.55 \mathrm{keV}$ ) —of the energetic levels involved in the radiation emission (Figure 11).

(b) The IR laser light $\rightarrow$ soft X-ray conversion efficiency increases/decreases by increasing/decreasing the laser energy (i.e., intensity) on the target according to a nonlinear, saturating, growing trend due to nonlinear processes involved in plasma emission (Figure 11).

(c) the IR laser light $\rightarrow$ soft X-ray conversion efficiency depends on the considered spectral region; that is, it varies by changing the soft $\mathrm{X}$-ray energy range.

(d) copper $(Z=29)$ and zinc $(Z=30)$ are the targets with the highest IR $\rightarrow$ soft X-ray conversion efficiency between those analyzed in the spectral range $(1.3-1.55) \mathrm{keV}$, that is respectively, $0.19 \% \leq \eta_{\mathrm{Cu}} \leq$ $0.39 \%$ and $0.17 \% \leq \eta_{\mathrm{Zn}} \leq 0.38 \%$.

(e) yttrium shows the lowest conversion efficiency between the investigated targets in the spectral range $(1.3-1.55) \mathrm{keV}$, that is $0.0068 \% \leq \eta_{\mathrm{Y}} \leq 0.023 \%$.
TABLe 9: Mean values of $V_{x}, E_{x}$, and $\eta$ for each considered target in the analyzed soft X-ray spectral region (1.3-1.55) keV.

\begin{tabular}{lccc}
\hline Atomic number $(Z)$ & $\left\langle V_{x}\right\rangle(\mathrm{V})$ & $\left\langle E_{x}\right\rangle(\mathrm{mJ})$ & $\langle\eta\rangle(\%)$ \\
\hline $12(\mathrm{Mg})$ & 22.0 & 11.8 & 0.16 \\
$22(\mathrm{Ti})$ & 9.3 & 5.0 & 0.071 \\
$26(\mathrm{Fe})$ & 16.8 & 9.1 & 0.13 \\
$29(\mathrm{Cu})$ & 40.1 & 21.6 & 0.30 \\
$30(\mathrm{Zn})$ & 40.5 & 21.8 & 0.30 \\
$39(\mathrm{Y})$ & 2.3 & 1.3 & 0.017 \\
\hline
\end{tabular}

(f) In the analyzed spectral region (1.3-1.55) $\mathrm{keV}$ and for the considered targets, the IR laser light $\rightarrow$ soft X-ray conversion efficiency $(\eta)$ is less than $1 \%$.

(g) The remaining laser energy not reflected by plasma and not converted to soft $\mathrm{X}$-rays is recovered as visible and ultraviolet radiation and, mainly, heat that increases the target temperature from environment one (about $25^{\circ} \mathrm{C}$ ) to that of plasma (around $10^{6 \circ} \mathrm{C}$ in our case).

(h) $V_{x}$ and $E_{x}$ take growing values by increasing the laser energy (i.e., intensity) according to a slightly saturating, quasilinear, growing trend (Figure 10).

(i) The average quantities $\left\langle V_{x}\right\rangle,\left\langle E_{x}\right\rangle$, and $\langle\eta\rangle$ are characterized by an irregular trend as a function of the atomic number $(Z)$ of the considered element (Figure 12 and Table 9).

(j) $\left\langle V_{x}\right\rangle,\left\langle E_{x}\right\rangle$ and $\langle\eta\rangle$ depend on the investigated target and spectral region. Studies to confirm the last part of this statement are in progress in our laboratory and, especially, for yttrium in the spectral range below $1 \mathrm{keV}$ (e.g., water window).

\section{Results: X-ray Spectroscopy}

$\mathrm{X}$-ray spectra are a fundamental diagnostic tool of local conditions in high-temperature plasmas such as those produced by ultrapowerful (up to PW-level) and ultrashort (of the order of fs) pulse laser sources able to give rise to a new regime of hot and dense plasmas [40-43].

Specifically, in these spectra, an essential role is covered by satellite lines in the vicinity of the parent spectral structures for obtaining useful information about plasma parameters (e.g., density, temperature, opacity, etc.), beside the task of the Stark broadening of spectral lines as sensitive probe of the electron density $[44,45]$ and that of $\mathrm{H}$ - and $\mathrm{He}-$ like resonance lines for measuring plasma polarization shifts $[46,47]$.

In addition, the satellites near to resonance lines can give rise to a significant effect distorting the broadened line profiles, so affecting the results of spectral analysis, as already shown in $[48,49]$. In this framework, we have recently carried out a series of experiments devoted to emphasize the importance of the line structure (e.g., satellites) in the X-ray spectra in order to develop a reliable diagnostics of plasma state estimating its main parameters. 


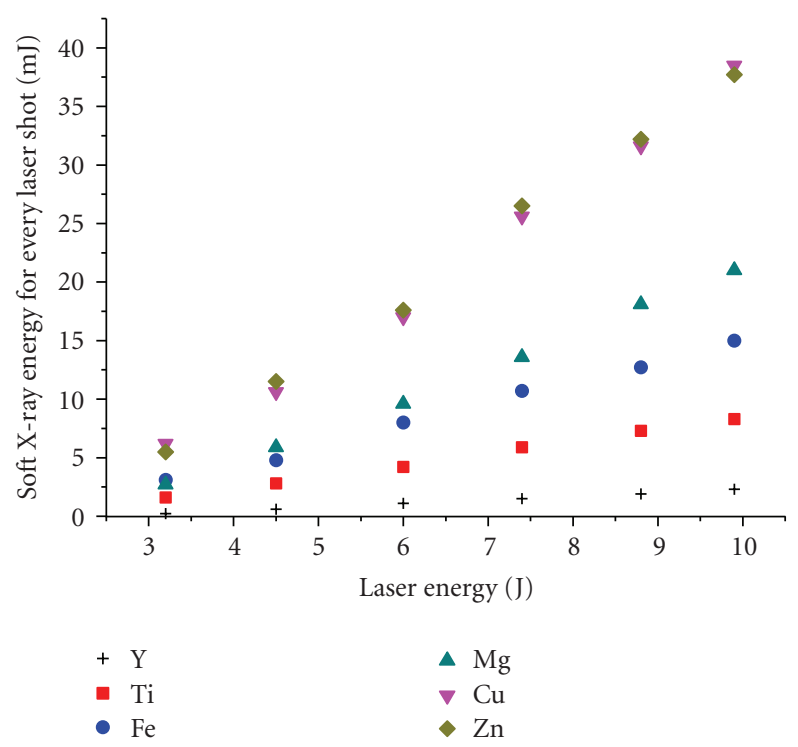

FIgUre 10: Soft X-ray energy emitted by the TVLPS—-detected by Quantrad PIN photodiode-as a function of laser energy for different solid targets in the spectral region (1.3-1.55) keV.

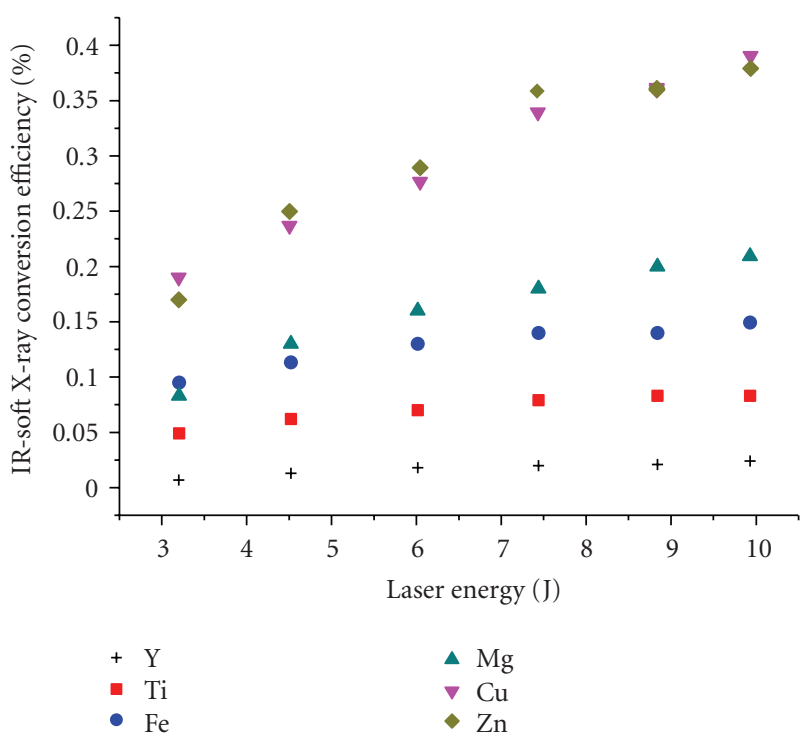

FIgURe 11: Conversion efficiency from infrared (IR) laser light to soft X-rays (spectral region $1.3-1.55 \mathrm{keV}$ ) as a function of laser energy for various solid targets obtained by the TVLPS.

More precisely, the experimental tests have especially concerned: (1) measures and identifications of the $L$-shell Rydberg series in the soft X-ray spectrum of Ne-like Zn XXI ions with particular reference to the high- $n$ dielectronic Rydberg satellites from Mg-like Zn XIX and Na-like Zn XX ions [18] and (2) measurements and modeling of the soft $\mathrm{X}$-ray emission of a barium plasma in the spectral range of high- $n$ Rydberg levels in a near Ni-like Ba XXIX ionization state [20].

A further brief analysis of soft X-ray spectra obtained for an iron plasma in a near Ne-like state is also reported

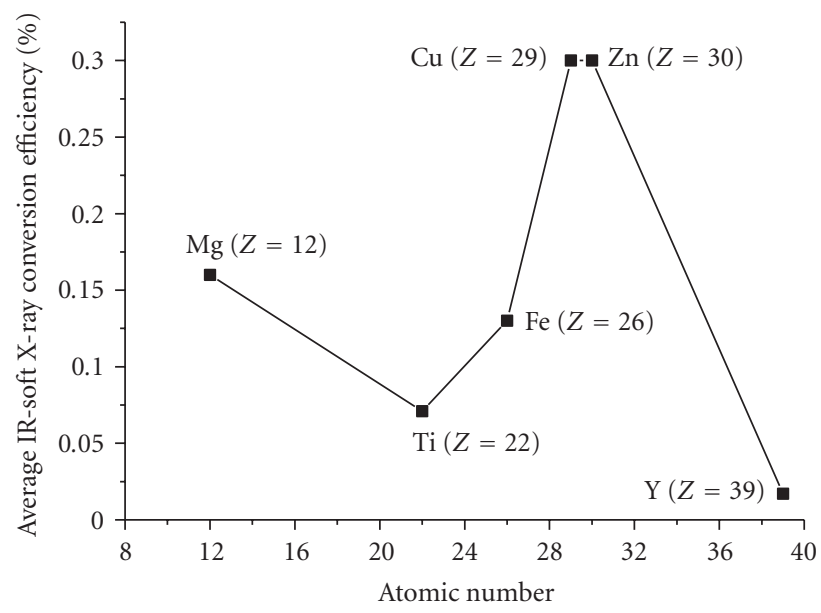

FIGURE 12: Average conversion efficiency from IR laser radiation to soft X-rays versus atomic number for different solid targets in the spectral region(1.3-1.55) keV.

with a special interest for: (a) the dielectronic $\mathrm{Mg}$-like $\mathrm{Fe}$ $\mathrm{XV}$ and Na-like Fe XVI satellite features near to the Nelike Fe XVII 3C and 3D parent resonance lines and (b) the dependence of the spectral features (e.g., line strengths) on the intensity of the illuminating laser strongly affecting the plasma temperature.

In the first case, it is worth to note that the Ne-like spectrum is the $L$-shell analog to the very useful He-like resonance lines and that the spectral identification of the Rydberg satellite structures to the Ne-like spectrum is an essential step for achieving a valid $L$-shell X-ray diagnostics in the high-temperature and high-density (close to that of a solid) matter, taking into account the relevance of the closedshell structure of the ions that enables one to attain a high stability over large temperature ranges [50].

Generally speaking, in the second case, the investigation of X-ray plasma spectra in a near Ni-like state is necessary for many applications involving high- $Z$ elements, such as physics for inertial confinement fusion [51] and the survey of impurity effects in magnetic confinement fusion [52].

In particular, in the specific case of our experiments in the field of X-ray plasma spectroscopy, the use of a laser source heating the matter, characterized by nanosecond (ns) pulse width and relatively low intensity compared to that achievable with ps (picosecond) or fs (femtosecond) shorter pulse laser facilities, is crucial for a precise identification of spectral lines on which a valid spectroscopic analysis is based and for the development of an effective diagnostics of plasma conditions, as, for example, occurs by using Ni-like spectra. In fact, a longer duration and a lower intensity of ns laser pulses guarantee the formation of an isothermal plasma with a negligible fraction of hot electrons, so no remarkable change is detected on the plasma ionization balance and spectral lines, as instead usually is observed for ps or fs laserplasma sources.

As a consequence, in this section, the attention will be strongly focused on the experimental and theoretical exploration of the Rydberg series of the Ni-like ion spectra 
from a heavy element (barium in our case). Such a study has been found particularly appropriate for an accurate analysis of the influence of the plasma parameters (bulk electron temperature, density, opacity, etc.) on the spectral line features (e.g., intensity).

In this study, two intriguing challenges have to be faced: (i) the accomplishment of a high spectral resolution in the obtained spectra for a precise identification of the many spectral lines arising from various ionization stages and (ii) the weak intensity of the lines.

The model calculations exploit the Los Alamos suite of atomic physics codes. Specifically, the CATS code [53] generates the significant atomic structure for the barium ions from Ge-like $\mathrm{Ba}$ XXV to Mn-like Ba XXXII (e.g., energy levels, oscillator strengths for proper transitions and plane-wave-Born electron-impact excitation cross-sections), producing hundreds/thousands of configurations per ion charge stage. The multipurpose GIPPER code $[54,55]$ is, instead, used for computing the autoionization rates and both the electron-impact ionization and the photoionization cross-sections necessary to the development of a collisional-radiative (CR) model. The ATOMIC code [56] permits the processing of the previous data in order to form a CR model and find a solution of the rate equations for the densities of state populations - then used to calculate the associated emission spectrum - in terms of input plasma parameters (temperature, density, etc.).

Finally, the MUTA option [57] in ATOMIC code enables one to construct theoretical spectra for reproducing the experimental ones. An explicit inclusion of strong spectral lines in a transition array and a statistical mixing of weaker lines are also provided by MUTA. Furthermore, an extra level-to-level CR model with configuration interactionyet with fewer configurations-is also used to verify the accuracy of MUTA results. In a few words, the CATS code is used to calculate the MUTA input data for the transition arrays, while the ATOMIC code merges the calculated configuration-average populations with the MUTA data for constructing the spectrum.

Ultimately, in the modeling calculations, the contributions due to various plasma ionization levels and the effects of the plasma temperature, density, and opacity on the investigated spectra are properly considered in an attempt to simulate the spectral lines experimentally observed.

In the experiments, the laser energy and the resulting intensity have been varied in the ranges (1-8) J and $\left(10^{11}-10^{12}\right) \mathrm{W} / \mathrm{cm}^{2}$, respectively. The doublet lens previously introduced in Section $2(d=5 \mathrm{~cm}, f=20 \mathrm{~cm})$ has been used for focusing the laser beam on a $\mathrm{BaF}_{2}$ target at a $45^{\circ}$ incidence angle in order to produce plasma. An estimated laser spot diameter of about $300 \mu \mathrm{m}$ has been attained at the focal plane then varied by defocusing up to a value of $500 \mu \mathrm{m}$.

A specific stepped rotating target configuration [19], consisting of two separate targets at a distance of (300800) $\mu \mathrm{m}$, has been also used in all experimental tests. More exactly, the first component was constituted by $\mathrm{BaF}_{2}$ (i.e., the investigated target), while the second part contained $\mathrm{Mg}$ or Al used to produce reference spectral lines.
The laser shots at each target have been done separately and, as already stated, the heights of the steps were (300800) $\mu \mathrm{m}$. Specifically, between laser shots, the targets have been moved in the plane perpendicular to laser beam, so permitting to obtain well-separated spectral lines of the investigated matter and of the reference materials by using the same spectrometer equipped with KODAK RAR 2492 film for recording the X-ray plasma emission spectra.

The procedure described in [58] has been adopted for developing, scanning by a microdensitometer and turning the film density into incident X-ray intensity. The used films have been placed in a suitable holder, adequately protected by two layers of $1 \mu \mathrm{m}$ polypropylene filters coated with $0.2 \mu \mathrm{m}$ aluminum sheets on both sides. Additional $2 \mu \mathrm{m}$ polypropylene layers have been used in order to stop the plasma debris directed towards the surface of spectrometer crystal.

The spectral line intensities have been properly corrected by taking into account the filter transmissions. At the same time, the reflectivity of spectrometer crystal has been assumed slowly varying over the investigated spectral range and not included in the calibrations.

So, in summary, we have been able to simultaneously acquire spatially resolved X-ray spectra of the high- $n(3<$ $n \leq 8)$ Rydberg line series for a near Ni-like Ba XXIX ion plasma in the spectral range (7.8-9.5) $\AA$ along with the calibration lines due to $\mathrm{H}$ - and $\mathrm{He}$-like $\mathrm{Mg}$ or $\mathrm{Al}$ ions by means of spherical crystal-based spectrometers (Section 2.2) with high spectral $(\lambda / \Delta \lambda>2000)$ and high spatial (30$50 \mu \mathrm{m})$ resolutions. More precisely, in the experiments two different spherically bent mica spectrometers have been used. The former was characterized by a $(15 \times 50) \mathrm{mm}^{2}$ large aperture crystal with a curvature radius of $R_{c}=$ $150 \mathrm{~mm}$, while the latter had a $(10 \times 30) \mathrm{mm}^{2}$-size crystal and a $R_{c}=100 \mathrm{~mm}$ curvature radius. The use of more spectrometers is fundamental for enhancing the spectral range and the spectral resolution during the observations. So, a theoretical spectral resolving power $\lambda / \Delta \lambda$ within the range $3000-5000$ is expected for the spectrographs, while an actual spectral resolution of $\lambda / \Delta \lambda \cong 2600$ is achievable under the experiment conditions.

This occurs because the measurement accuracy is essentially due to the line profile widths - mainly determined by Doppler, natural, and instrumental broadening $\Delta_{\mathrm{FWHM}}-$ and spectral line blending, in a framework where the wavelengths of the used reference lines are known with high precision (better than $0.22 \mathrm{~m} \AA$ ), as already demonstrated in [18].

Concerning the accuracy of the spectral lines, a distinction between symmetric and asymmetric line profiles is necessary. Specifically, line center positions are measurable with a precision better then $\left(0.1 \leq \Delta_{\text {FWHM }} \leq 0.5\right) \mathrm{m} \AA$ for symmetric spectral profiles, while a worse accuracy up to two or three times is observable for asymmetric blended lines. Moreover, generally speaking, the position of line center can be regularly shifted because of Doppler effect owing to plasma expansion. However, in the specific case of our experiments, the line shift due to systematic Doppler effect is smaller than $0.26 \mathrm{~m} \AA$, since the spectra have been recorded at circa $85^{\circ}-90^{\circ}$ angles relative to the direction of plasma expansion, though a plasma speed of $10^{7} \mathrm{~cm} / \mathrm{s}$ 


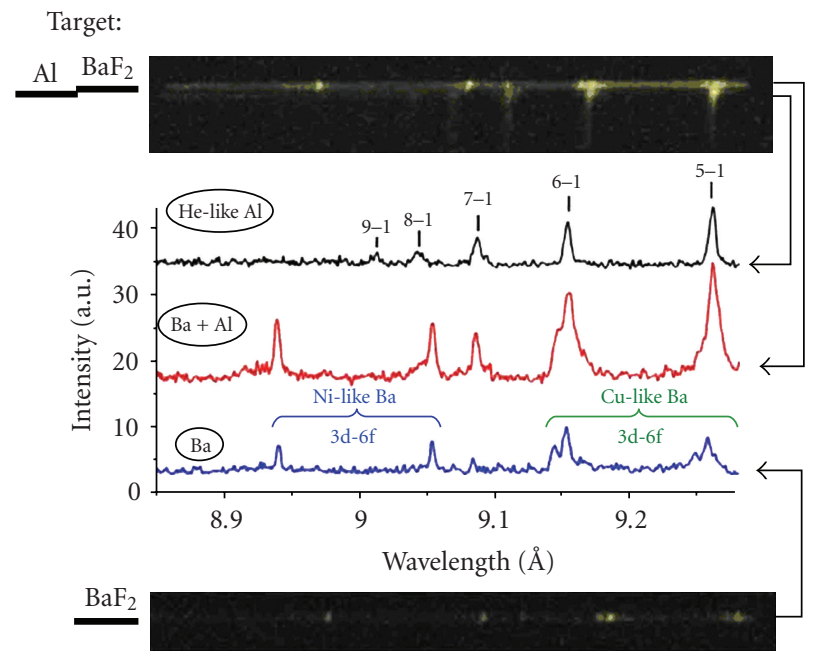

(a)

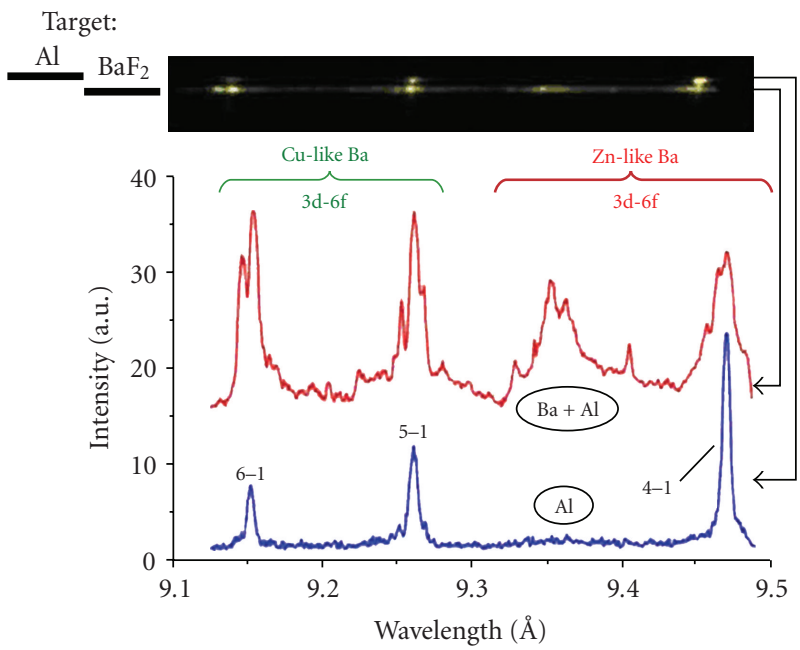

(b)

FIGURE 13: Soft X-ray emission spectra of dense and hot $\mathrm{BaF}_{2}$ plasma and $\mathrm{Al}$ reference spectra obtained by using the TVLPS equipped with the large aperture spherically bent mica spectrometer $\left(R_{c}=150 \mathrm{~mm}\right)$ and stepped rotating targets. The investigated spectral regions vary in

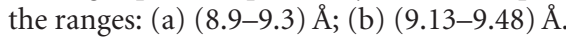

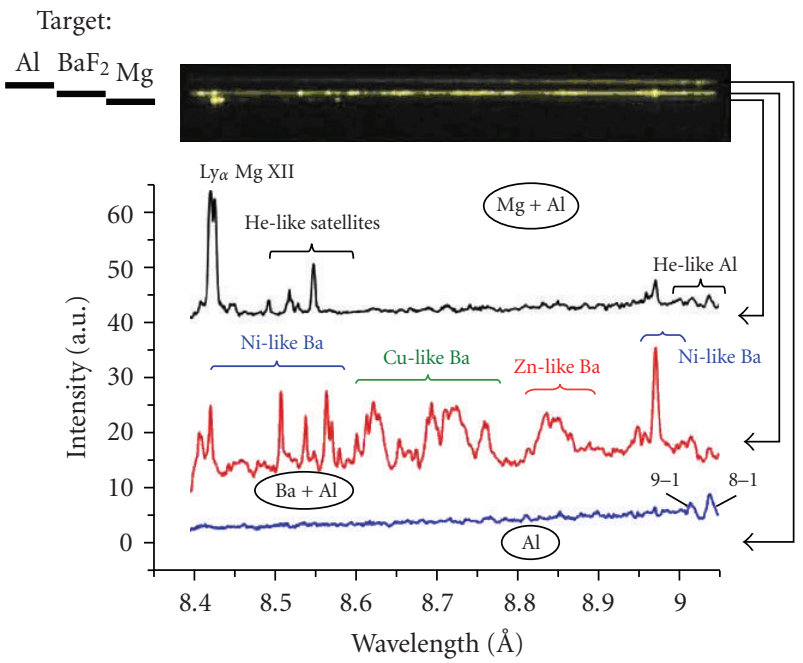

(a)

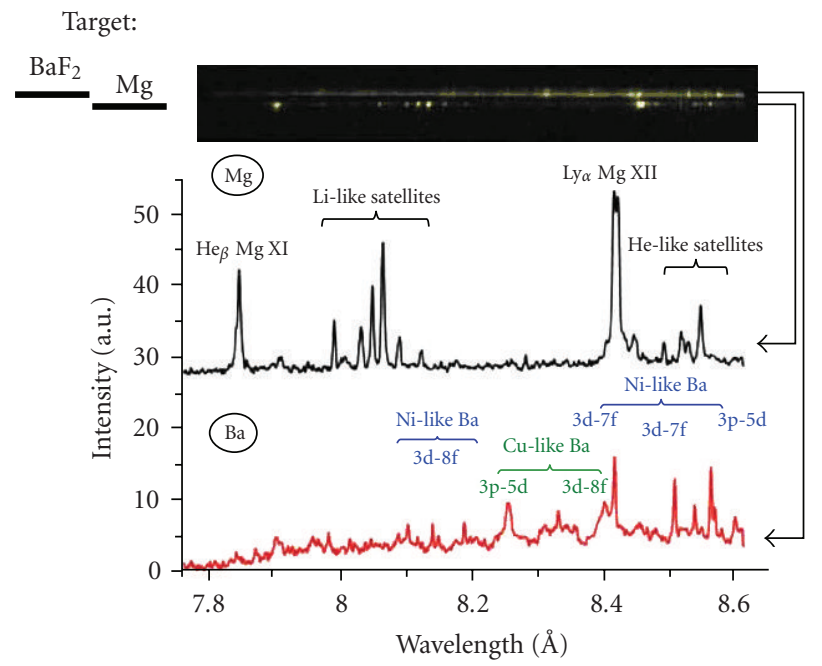

(b)

FIGURE 14: Soft X-ray emission spectra of dense and hot $\mathrm{BaF}_{2}$ plasma and reference spectra of $\mathrm{Mg}$ and $\mathrm{Al}$ obtained by using the TVLPS equipped with the $R_{c}=100 \mathrm{~mm}$ spherically bent mica spectrometer and step rotating targets. The investigated spectral regions are

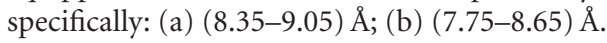

is observable. As a result, considering that calibration lines are also shifted because of the reference ion motion, the Doppler shift can be neglected, since it is from three to ten times smaller than the expected measurement accuracy. So, a total accuracy of about $(0.5-1.5) \mathrm{m} \AA$ is effectively obtained in our measurements of the spectral line wavelengths. The recorded spectra of $\mathrm{Ba}$ plasma are characterized by some overlapping spectral regions due to the use of high-resolution spherically bent spectrometers able to investigate a $(0.3-$ $0.7) \AA$ range in a single laser shot. Each region, however, contains a few reference lines with known wavelengths, especially corresponding to the spectral lines of He-like $\mathrm{Mg}$ $\mathrm{XI}$ and $\mathrm{Al}$ XII.

Such experimental spectra are shown in Figures 13 and 14 , while their comparison with theoretical ones, modeled by a detailed collisional-radiative approach based on ATOMIC MUTA calculations, is reported in Figures 15 and 16. In all comparisons line-to-continuum ratio is an adjustable parameter.

The results depicted in Figure 13 have been obtained with two laser shots by using the $R_{c}=150 \mathrm{~mm}$ large aperture spectrometer and considering a stepped rotating target 


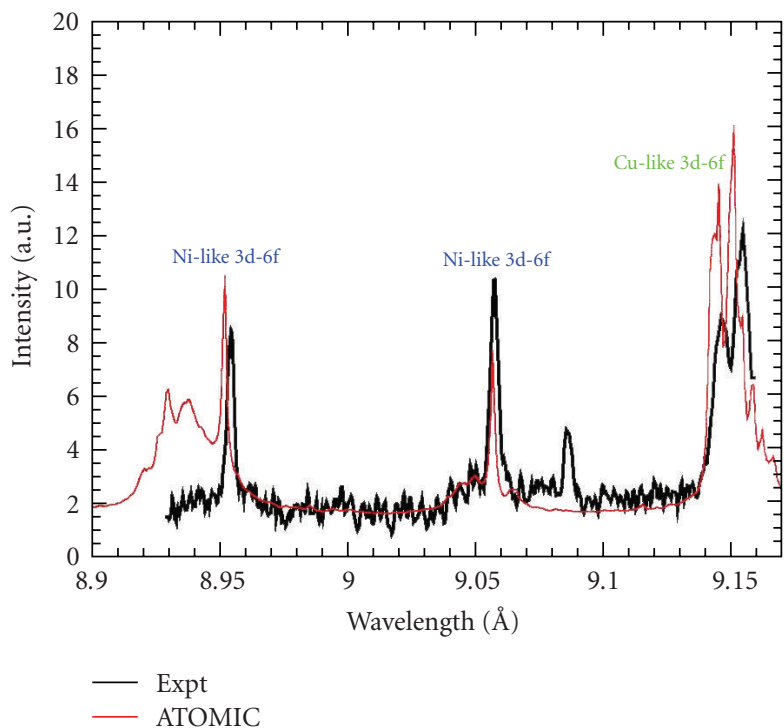

(a)

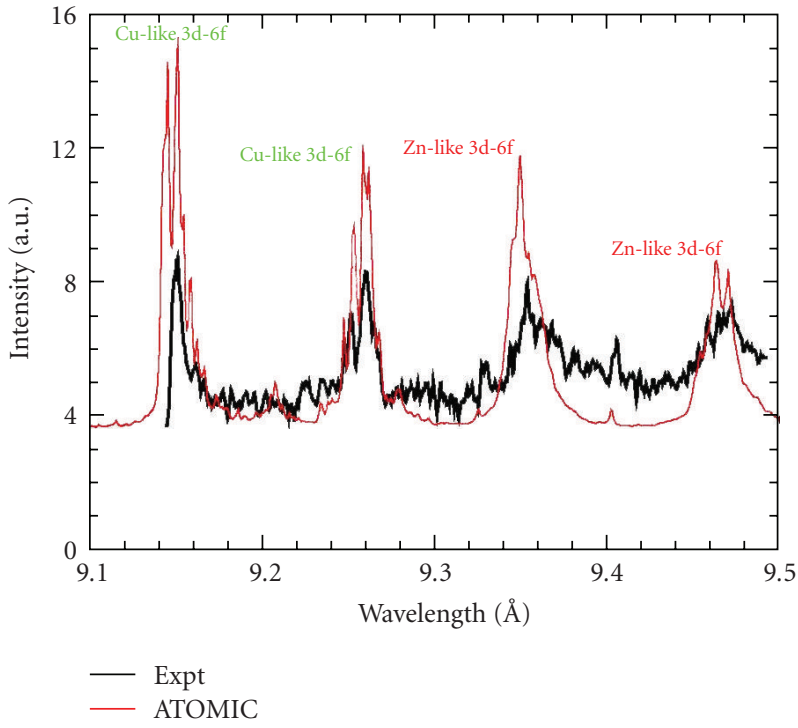

(b)

FIGURE 15: Comparison between experimental spectra, captured by the large aperture spherical mica crystal-based spectrometer with curvature radius of $R_{c}=150 \mathrm{~mm}$, and the theoretical spectra obtained by the ATOMIC MUTA model calculations performed at the following plasma conditions: (a) $T_{e}=220 \mathrm{eV}$ (electron temperature); $N_{e}=5 \times 10^{20} \mathrm{~cm}^{-3}$ (electron density); (b) $T_{e}=300 \mathrm{eV}, N_{e}=3 \times 10^{20} \mathrm{~cm}^{-3}$, size $=100 \mu \mathrm{m}$. Note that the investigated spectral region falls into the soft X-ray range (8.9-9.5) $\AA$ and that only in the case (b) the theoretical calculations include the effect of a finite plasma size (i.e., $100 \mu \mathrm{m}$ ) on the X-ray spectra. An identification of the main line transitions is finally reported.

composed by $\mathrm{Al}$ and $\mathrm{Ba}$. The outcomes of Figure 14 have been always attained with two laser shots but using the $R_{c}=100 \mathrm{~mm}$ spectrograph for two different target setups: the former employing $\mathrm{Al}, \mathrm{Ba}$, and $\mathrm{Mg}$ stepped targets and the latter only $\mathrm{Ba}$ and $\mathrm{Mg}$ stepped targets.

In Figure 15, instead, the spectral lines experimentally acquired by using the $R_{c}=150 \mathrm{~mm}$ spectrometer are compared to those obtained by the ATOMIC MUTA calculations in the soft X-ray spectral range (8.9-9.5) $\AA$ assuming various plasma parameters explicitly indicated in the caption of the figure (panels (a) and (b)). Specifically, the panel (a) shows a comparison within the wavelength range (8.9-9.15) $\AA$ between the theoretical results and the experimental ones presented in Figure 13, panel (a). A similar comparison in the spectral range (9.15-9.5) $\AA$ is also reported in the panel (b) where the calculated spectral features are compared to those experimentally measured, previously illustrated in Figure 13, panel (b). Moreover, the more prominent line transitions are also identified.

A good agreement between the synthetic spectral lines and the measured ones is attained for the spectra of Figure 15. In fact, the model calculations permit to fairly well simulate the observed main spectral features (line positions and intensities) that, in the investigated spectral range, are all 3d-6f transitions in Ni-like, Cu-like, and $\mathrm{Zn}$-like ions of a barium plasma.

The experimental spectral lines are not well reproduced by the calculations only in some cases. For example, this occurs for a wavelength of $9.085 \AA$ due to a $3 \mathrm{~d}-6 \mathrm{f}$ line transition in Ga-like Ba ions that, though included in the theory, appears extremely weak under the considered plasma conditions (see Figure 15), since the population of this ionic charge state is very small. As a result of such comparisons, one infers that a noneven temperature distribution may exist in the laser induced plasma since lower temperatures are necessary to increase the ion stage population.

Another instance where the spectral features theoretically expected by the ATOMIC MUTA calculations are not retrieved in the measured spectra is represented by the satellites up to the $3 \mathrm{~d}$ - $6 \mathrm{f} \mathrm{Ni}$-like line at $8.95 \AA$. In fact, these lines are placed at the edge of the experimental spectrum, and, consequently, they are characterized by an intensity too low.

On the other hand such spectral lines are actually observed-yet with an intensity slightly less than that of the calculated lines-in the recorded spectrum reported in the panel (a) of Figure 16.

Generally speaking, in this figure, the experimental spectra, obtained by the $R_{c}=100 \mathrm{~mm}$ spectrometer, are compared to those simulated by the ATOMIC MUTA code in the (7.8-9) $\AA$ soft X-ray spectral range considering different plasma conditions properly emphasized in the caption of the figure (panels (a) and (b)).

More precisely, the panel (a) newly shows a comparison within the wavelength range (8.4-9) $\AA$ between the results expected by theory and those experimentally found, previously presented in the panel (a) of Figure 14. At the same time, the panel (b) of Figure 16 compares the modeling calculations to the measurements reported in Figure 14, panel (b), in the spectral range (7.8-8.6) $\AA$. 


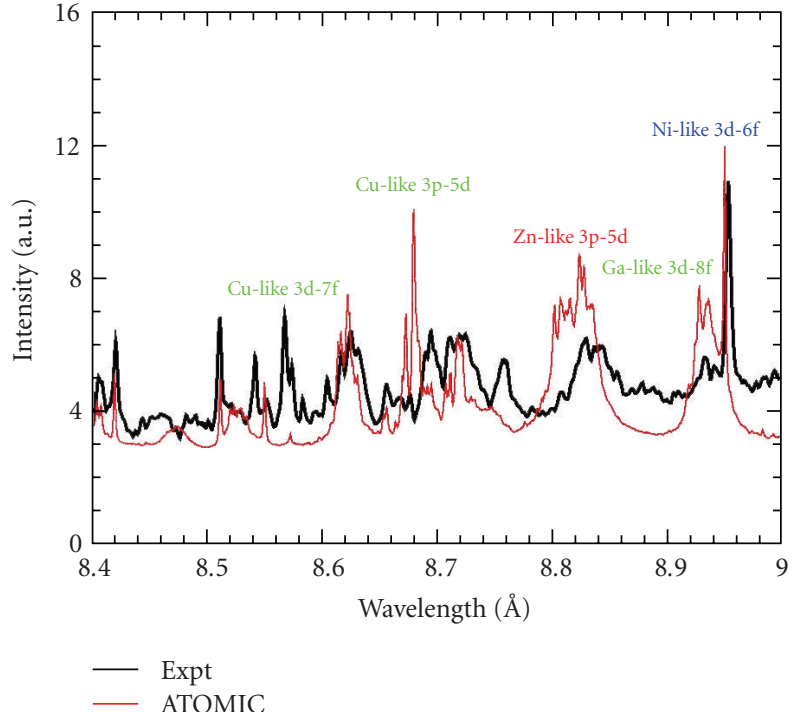

(a)

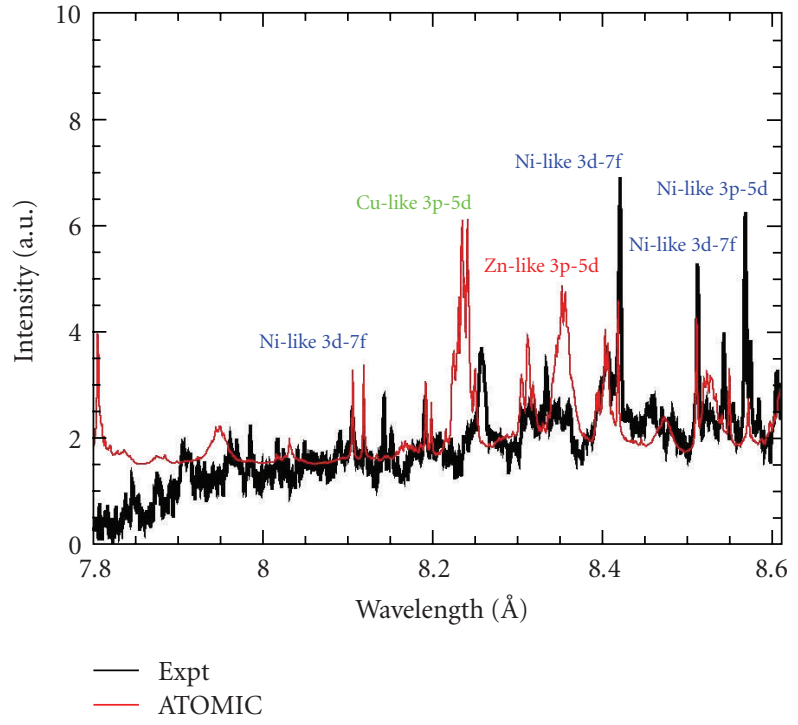

(b)

Figure 16: Comparison between experimental spectra, captured by the spherical mica crystal-based spectrometer with $R_{c}=100 \mathrm{~mm}$ curvature radius, and the spectra modeled by the ATOMIC MUTA calculations carried out at the following plasma conditions: (a) $T_{e}=220 \mathrm{eV}$ (electron temperature); $N_{e}=5 \times 10^{20} \mathrm{~cm}^{-3}$ (electron density); (b) $T_{e}=250 \mathrm{eV}, N_{e}=5 \times 10^{20} \mathrm{~cm}^{-3}$, size $=100 \mu \mathrm{m}$. Note that the surveyed spectral region falls into the soft X-ray range (7.8-9) $\AA$ and that only in the case (b) the model calculations include the effect of a finite plasma size (i.e., $100 \mu \mathrm{m}$ ) on the X-ray spectra. An identification of the main spectral features is finally reported.

In addition, the more important line transitions are recognized and a quite satisfactory agreement between experimental and theoretical results is again achieved, especially for the spectral line positions considering the extreme complexity of the electronic structure of the ion charge stages undergoing transitions. Nevertheless, in some cases, the intensities of synthetic lines are different with respect to the measured ones. These gaps could be due to temperature and/or density gradients in the plasma responsible for the intensity variations of the observed lines.

The main plasma parameters, considered in Figures 15 and 16, have been evaluated after performing a series of calculations over a reasonable range of values in an attempt to find plasma conditions that better permit to reproduce the experimental spectra.

Some examples of these calculations are reported in Figures 17 and 18 [20]. Specifically, the synthetic spectra obtained by the ATOMIC MUTA code for plasma electron temperatures varying in the range $(150-300) \mathrm{eV}$ and for a constant plasma electron density of $N_{e}=5 \times 10^{20} \mathrm{~cm}^{-3}$ are shown in Figure 17. Under these conditions, the plasma average ionization is comprised between 25.5 (at $150 \mathrm{eV}$ ) and 27.2 (at $300 \mathrm{eV})$. As a consequence, the same ion charge stages are essentially responsible for the plasma emission at all temperatures.

In Figure 18, instead, the theoretical ATOMIC MUTA spectra of a barium plasma, calculated at a $250 \mathrm{eV}$-fixed electron temperature and at an electron density varying from $10^{20} \mathrm{~cm}^{-3}$ to $10^{21} \mathrm{~cm}^{-3}$, are illustrated.

Another instance of the model calculations is reported in Figure 19 [20], where the plasma electron temperature

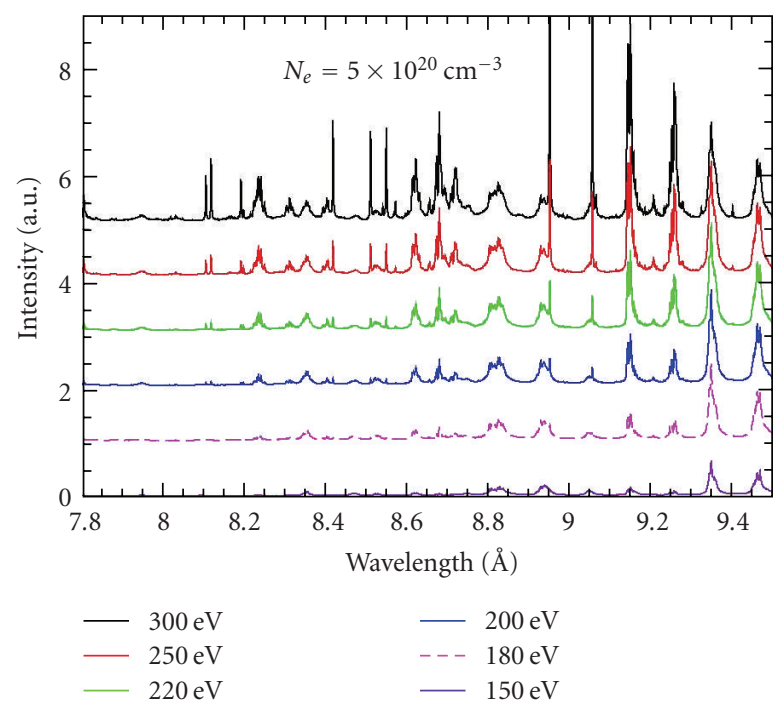

FIgURE 17: Theoretical spectra obtained by the ATOMIC MUTA calculations carried out at various plasma electron temperatures and a fixed electron density of $N_{e}=5 \times 10^{20} \mathrm{~cm}^{-3}$. Each spectrum has been translated on the $y$ axis for a better clarity in the arrangement of the results (see [20]).

and density are maintained at a fixed value $\left(T_{e}=250 \mathrm{eV}\right.$ and $N_{e}=5 \times 10^{20} \mathrm{~cm}^{-3}$, resp.), while the plasma size $L$ is varied in the range $(0-1000) \mu \mathrm{m}$ for monitoring the effect of the plasma dimension (i.e., opacity) on the spectral lines, including this effect in an approximate way by means of the escape factors $\varepsilon[29,59-61]$. 


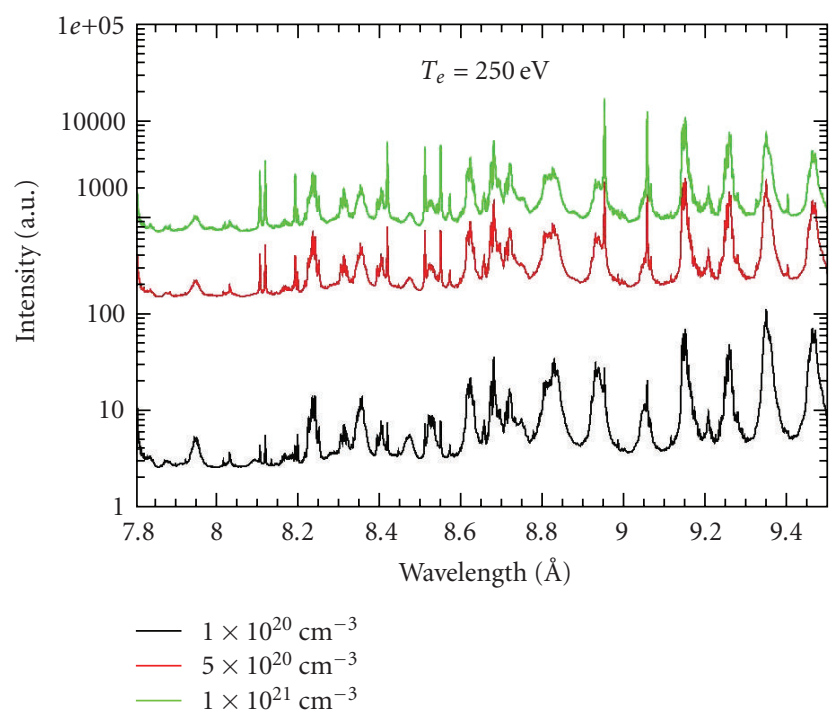

FIgUre 18: Theoretical spectra expected by the ATOMIC MUTA calculations performed at a fixed plasma electron temperature of $250 \mathrm{eV}$ and at various electron densities (see [20]).

In fact, neglecting the stimulated emission, the plasma opacity is strictly related to the optical depth $\tau(v)$ that, for a spectral line of frequency $v$, is given by the product between the line opacity and the number density $N_{l}$ of absorbing ions, integrated over the line of sight through the plasma.

As a result, a simple linear relationship between optical depth and size of a plasma is derived according to

$$
\tau(\nu)=\kappa(\nu) N_{l} L .
$$

Here, $\kappa(v)$ is a proportionality factor depending on $v$ and $\varepsilon$, whose expression takes formally the form [18]

$$
\begin{aligned}
\kappa(\nu) & =\frac{e^{2}}{m c^{2}} f_{l, u} \sqrt{\frac{\pi M c^{2}}{2 k_{B} T_{i}}} \lambda \phi(\nu) \\
& =\left(1.53 \times 10^{-24}\right) \frac{g_{u} A_{\mathrm{eff}}}{g_{l} \varepsilon(\Delta E)^{3}} \sqrt{\frac{\mu}{T_{i}}} \phi(\nu),
\end{aligned}
$$

where $m$ and $e$ denote the electron mass and charge, respectively, $c$ is the vacuum light speed, $f_{l, u}$ the absorption oscillator strength for the considered line, $M$ and $T_{i}$ - usually assumed equal to $T_{e}$-are the mass and temperature of absorbing ion, respectively, $k_{B}$ is the Boltzmann constant, $\lambda$ the transition wavelength, and $\phi(\nu)$ the normalized line profile. The $g$ 's parameters, instead, denote the statistical weights of the levels involving in the line transition, $A_{\text {eff }}$ is the effective transition rate, $\mu$ the ion mass in units of proton mass and, finally, $\Delta E$ the transition energy in hartrees.

From Figure 19, one establishes that most of the calculated spectra are rather insensible to the plasma dimensions though a few spectral lines such as those at $8.95 \AA$ and $9.05 \AA$, are sensitively suppressed by including size effects. On the contrary, the synthetic spectra of Figures 17 and 18 allow to conclude that the spectral features are greatly influenced by electron temperature and density of the generated plasma.

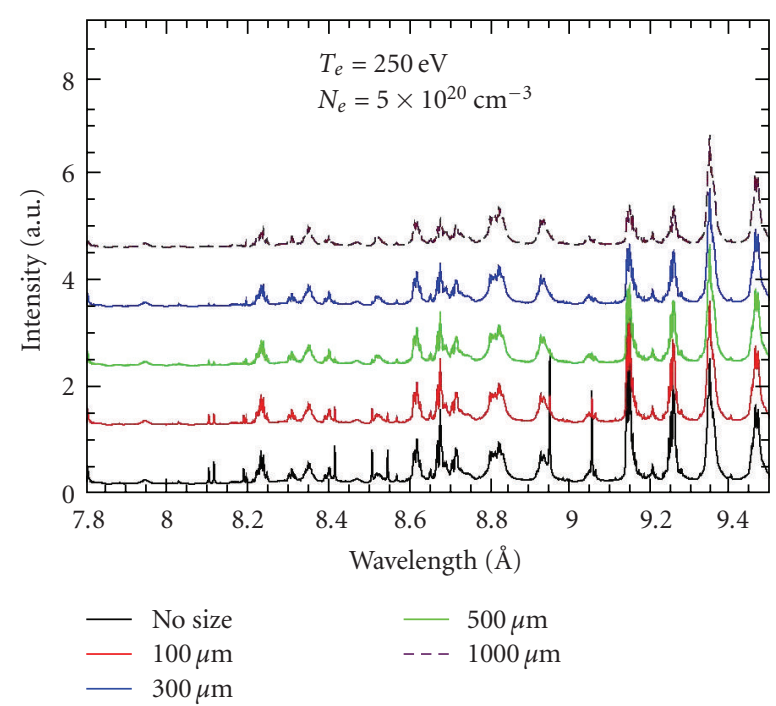

FIgURE 19: Theoretical spectra calculated by the ATOMIC MUTA code for constant plasma electron temperature $\left(T_{e}=250 \mathrm{eV}\right)$ and density $\left(N_{e}=5 \times 10^{20} \mathrm{~cm}^{-3}\right)$, considering various plasma sizes ranging between 0 (no size) and $1000 \mu \mathrm{m}$. Each spectrum has been shifted on the $y$ axis for a better visual clarity of the obtained results (see [20]).

In summary, by taking into account all the theoretical results reported from Figures 17 to 19 and by their comparison with the experimental outcomes (see specifically Figures 15 and 16), it is possible to conclude that the conditions of the barium plasma experimentally produced by the nanosecond (ns) Tor Vergata laser-plasma facility are well reproduced by assuming an isothermal and spherical plasma with an electron temperature $T_{e}=T_{i} \cong 250 \mathrm{eV}$ and density $N_{e} \cong 5 \times 10^{20} \mathrm{~cm}^{-3}$, with a prominent size of some hundreds of $\mu \mathrm{m}$ (e.g., $100 \mu \mathrm{m}$, optically opaque plasma) and with an average ionization balance equal to 26.8 , evaluated via the ATOMIC MUTA calculations and confirmed by the fine-structure computations [20]. Finally, no hot electrons are observed [29].

In fact, for example, it is only at an electron temperature close to $250 \mathrm{eV}$ that all the spectral lines due to transitions from barium ions have a practically equal intensity compared to that found in the measured spectra of Figures 13 and 14. On the other hand, considering higher temperatures, the lines around $9 \AA$ are not well simulated, since they are too intense, while at lower temperatures the low-wavelength lines are not reproduced.

As last theoretical study, in Figure 20 [20], the main contributions to the $\mathrm{Ba}$ spectrum due to the five ions charge stages listed in the figure are shown for a $250 \mathrm{eV}$ electron temperature and a $5 \times 10^{20} \mathrm{~cm}^{-3}$ electron density of barium plasma. Specifically, the ion charge stages that mainly contribute to the spectra in the wavelength range (7.8-9.5) $\AA$ extend from Ga-like Ba XXVI to Co-like Ba XXX.

In addition, in this figure, a classification of the predominant spectral lines, involving an electron moving from an excited configuration into that identified by $n=3$, is also reported. Within this framework, the main contributionsunder the considered plasma parameters and the investigated 


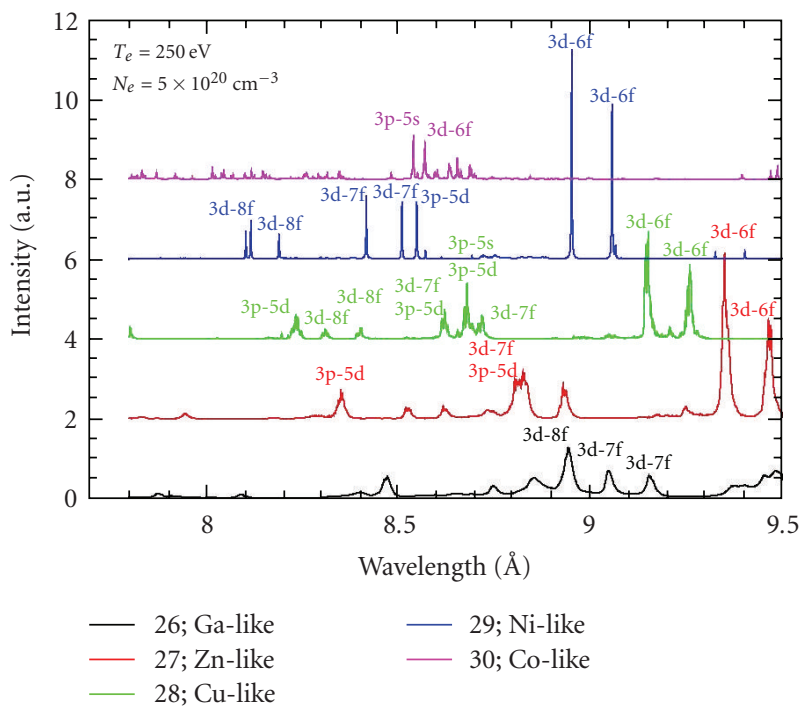

Figure 20: Theoretical spectra simulated by the ATOMIC MUTA code in the wavelength range (7.8-9.5) $\AA$ for plasma electron temperature and density of $250 \mathrm{eV}$ and $5 \times 10^{20} \mathrm{~cm}^{-3}$, respectively. The spectral contributions due to the five main charge stages of the ions under these barium plasma conditions are shown along with the main line transitions opportunely identified. Each spectrum has been arbitrarily scaled on the $y$ axis for a clearer presentation of the results (see [20]).

spectral range-come from the transitions $(3 \mathrm{p}, 3 \mathrm{~d})-n l$ with $n=5-8$ and $l=0-3$. As a result, one infers that the $\mathrm{Zn}$-like $\mathrm{Ba}$ XXVII, Cu-like Ba XXVIII, and Ni-like Ba XXIX ion stages contribute mainly to the spectral line emission.

A significant hot new is that the spectroscopy studies for highly excited transitions so far presented belong to the narrow family of the information concerning barium plasma emission spectra in the region of soft X-rays.

In fact, very few previous investigations are actually available for a comparison with the obtained spectral lines. More precisely, the only preceding studies in this field that we know are those reported in $[62,63]$, where spectral line positions have been explored in the range (8-13.5) $\AA$ and, especially, within the wavelength region (9.1-9.36) $\AA$.

However, it is worth to emphasize that, in the considered wavelength range, our results are in very good agreement with those shown in $[62,63]$.

Similar spectroscopic surveys have been previously made in the X-ray field for plasmas composed by a reasonable fraction of highly charged copper (from F-like $\mathrm{Cu}$ XXI to Olike $\mathrm{Cu}$ XXII) and zinc (from Mg-like Zn XIX to Na-like Zn $\mathrm{XX}$ ) ions in a near Ne-like state involving the L-shell and Mshell $[16,18,29]$.

These studies have been carried out by using the ns TVLPS along with other laser-plasma facilities, such as the picosecond (ps) Neodym facility [64] at the Central Research Institute of Machine Building near Moscow, the ns Hercules facility [18] at the ENEA research center in Frascati near Rome, and the femtosecond (fs) Saclay 10-TW UHI10 facility [65] near Paris.
The investigations have been mainly focused on: (a) the role covered by satellites in the vicinity of the principal Ne-like resonance lines on the observed spectra, especially in an attempt to establish the plasma conditions generating the spectra and (b) the effects of the opacity and hot electrons on the spectral lines emitted by plasmas with highly charged ions (e.g., Ne-, F-, and O-like ions).

Specifically, concerning the second case (b), we only emphasize that, focusing our attention especially on the TVLPS, opacity effects-treated in terms of escape factorsare necessary to get the correct relative intensities of high$n(n=5,6)$ emission features of a laser-induced plasma (e.g., copper plasma in a near Ne-like $\mathrm{Cu}$ XX ion state [29]), that is, for obtaining a good agreement between theoretical and experimental spectra. The influence of the hot electrons, instead, can be neglected in this case, so confirming the results previously exposed in this section for a barium plasma.

On the contrary, in the following a major attention is devoted to main results attained by the TVLPS pertaining to the point (a), that is, the recording and the analysis of the spectral features of the $\mathrm{Na}$ - and $\mathrm{Mg}$-like satellite lines observed for high- $n(n=4,5)$ Rydberg transitions in the Ne-like Zn XXI ion spectra in an attempt to obtain a valid modeling of these spectra [18].

In fact, for avoiding that plasma electron temperature and density are overestimated when resonance lines are broad, accurate high-spectral-resolution measurements should be done in order to evaluate the Rydberg satellite nature, taking into account that spectral features are strongly depending on the plasma conditions (temperature, density, opacity, and hot electron fraction), as clearly highlighted in [66] for the spectral lines of L-shell Mo ion plasma.

On the other hand, the Rydberg satellites can affect the shape of the parent lines such as the main Ne-like resonance ones though it is usually very intricate to measure the influence of such satellites to the Ne-like lines, as especially occurs for the so-called 3C and 3D lines. Generally speaking, the satellites radiated from Rydberg $\mathrm{Na}$ - and $\mathrm{Mg}$ like autoionizing levels are characterized by two different decay channels: the former provides spectral lines near 3C and $3 \mathrm{D}$ ones, while the latter gives rise to satellites near the Rydberg Ne-like lines.

Fortunately, the satellite lines due to the second decay channel spectrally can be very well resolved and, thus, recorded, modeled, and used to allow the determination of Rydberg satellite intensity falling into the vicinity of the investigated Ne-like lines (e.g., 4C, 4D, 5C, 5D, etc., as particularly shown in [18]).

The more interesting spectroscopic results that we have obtained in this framework, characterized by high spectral $(\lambda / \Delta \lambda \cong 3000)$ and spatial $(20-50 \mu \mathrm{m})$ resolution, are shown from Figure 21 to Figure 25, after proceeding as before for the spectroscopy studies of the barium plasma, that is, for example, (1) use of stepped rotating target, containing zinc (investigated target) and $\mathrm{Mg}$ or Al (calibration target for producing well-known reference spectral lines), (2) covering of the studied wavelength range with various spherically bent spectrometers for 


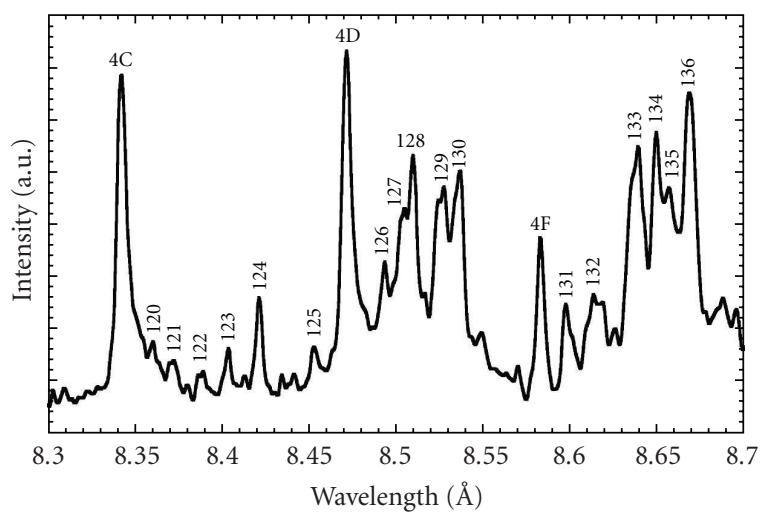

FIGURE 21: Soft X-ray spectrum recorded in the vicinity of the $n=$ 4 Ne-like Zn XXI ion resonance lines with noticeable dielectronic Rydberg Zn XIX (Mg-like) and Zn XX (Na-like) satellites obtained in the wavelength range (8.3-8.7) $\AA$ by using the ns TVLPS. Note that all the spectral lines are well identified, including the parent resonance lines $(4 \mathrm{C}, 4 \mathrm{D}$ and $4 \mathrm{~F}$ ) of the satellite ones (the remaining spectral features).

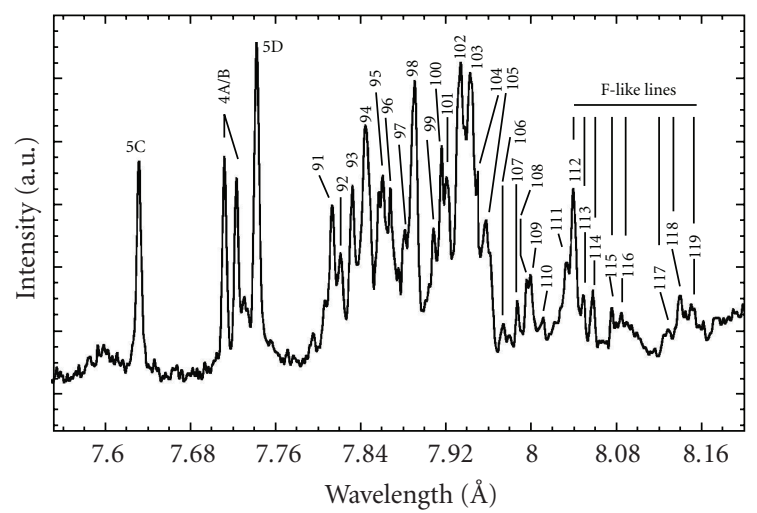

Figure 22: Measured soft X-ray spectrum showing the $n=5$ (5C and 5D) Ne-like Zn XXI ion resonance lines, some F-like Zn XXII ion spectral features - properly marked on the right edge - and the strong dielectronic Rydberg Zn XIX (Mg-like) and Zn XX (Nalike) satellites for a zinc plasma in a near Ne-like Zn XXI ion state. The spectrum has been attained within the wavelength range (7.6$8.16) \AA$ by using the ns TVLPS. Note that all the spectral lines are well identified along with the two $4 \mathrm{~A}$ and $4 \mathrm{~B}(n=4)$ Ne-like $\mathrm{Zn}$ XXI resonance lines.

allowing a much higher instrumental spectral resolution during the acquisition of spatially-resolved X-ray spectra, (3) recording of these spectra on KODAK RAR 2492 films, (4) possibility of covering a wavelength range of $(0.3-0.7) \AA$ in a single shot thanks to high resolution of spectrographs, (5) overall accuracy of wavelength measurements comprised in the range $(0.5-1.5) \mathrm{m} \AA$, and so forth.

Specifically, an example of soft X-ray emission spectrum, obtained by the ns TVLPS in the spectral range (8.3-8.7) $\AA$ for a zinc plasma in a near Ne-like Zn XXI ion state, is illustrated in Figure 21. It clearly shows some $n=4$

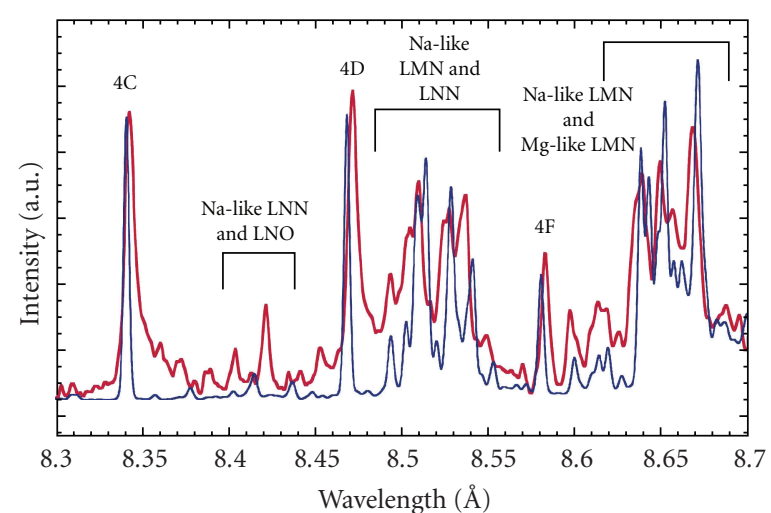

FIGURE 23: Comparison between soft X-ray spectra measured (blue) and calculated by CR model (pink) in the vicinity of the $n=4$ Ne-like Zn XXI resonance lines (4C, 4D, and 4F) with prominent dielectronic Rydberg Zn XIX (Mg-like) and Zn XX (Nalike) satellites in the spectral range (8.3-8.7) $\AA$. Note that optical depths with $L=200 \mu \mathrm{m}$ have been considered, especially for the resonance (i.e., Ne-like) lines.

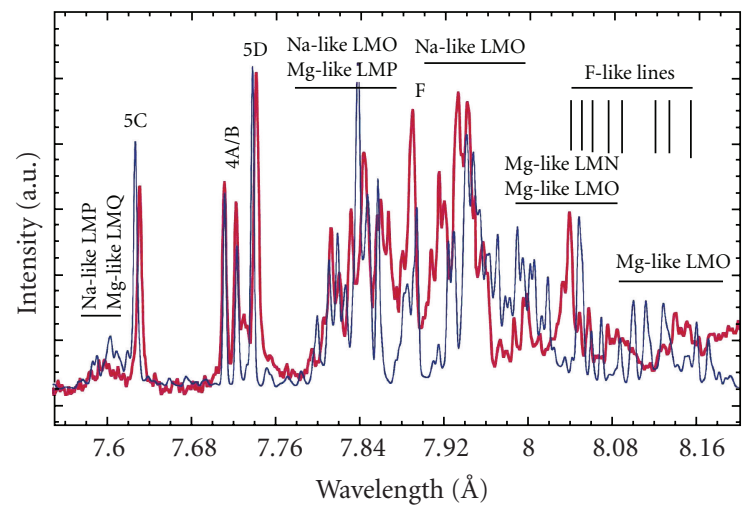

FIGURE 24: Comparison between soft X-ray spectra measured (blue) and calculated by CR model (pink) in the vicinity of the $n=5 \mathrm{Ne}$ like $\mathrm{Zn}$ XXI resonance lines (5C and 5D) with strong dielectronic Rydberg Zn XIX (Mg-like) and Zn XX (Na-like) satellites in the spectral range (7.6-8.16) $\AA$. Note that some F-like Zn XXII ion spectral features, properly indicated, are also showed and that optical depths with $L=50 \mu \mathrm{m}$ have been considered.

resonance lines due to Ne-like Zn XXI ions (i.e., explicitly, the $4 \mathrm{C}, 4 \mathrm{D}$, and $4 \mathrm{~F}$ lines) along with the pronounced dielectronic Rydberg Zn XIX (Mg-like) and Zn XX (Na-like) satellites in the vicinity of the parent resonance lines, corresponding to the remaining spectral features. A detailed identification of the spectral lines, strongly influenced by large optical depths because of the opacity of the generated plasma, is also reported for the considered spectral range.

Another instance of $\mathrm{Zn}$ plasma emission spectrum in the soft X-ray wavelength range (7.6-8.16) $\AA$, recorded in the vicinity of the Ne-like Zn XXI ion state, is depicted in Figure 22. The latter provides a representation of: (1) a few $n=5$ (i.e., 5C and 5D) Ne-like Zn XXI ion resonance lines, (2) some F-like Zn XXII ion spectral features, properly 


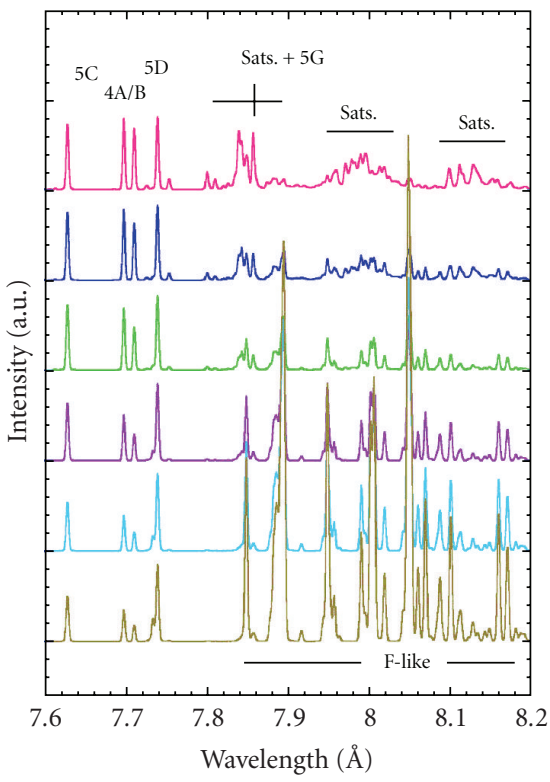

(a)

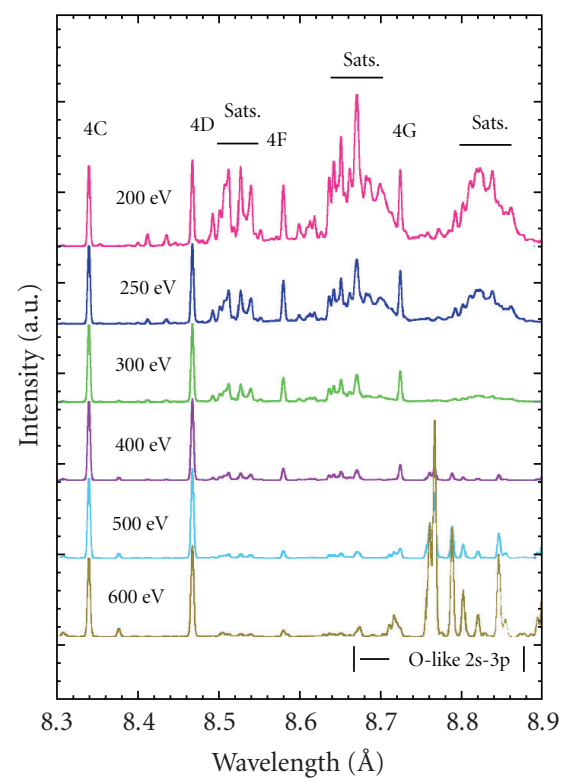

(b)

FIGURE 25: Simulated soft X-ray spectra in the vicinity of the $n=4$ (b) and $n=5$ (a) Ne-like Zn XXI resonance lines with noticeable dielectronic Rydberg satellites for various electron temperatures with a fixed plasma electron density $\left(N_{e}=10^{21} \mathrm{~cm}^{-3}\right)$ and an optical depth calculated for a constant $L=200 \mu \mathrm{m}$ plasma size. The spectra are calculated in the wavelength ranges (7.6-8.2) $\AA$ (a) and (8.3-8.9) $\AA$ (b).

emphasized on the right edge and corresponding to 112-119 spectral lines, and (3) the strong dielectronic Rydberg $\mathrm{Zn}$ XIX (Mg-like) and Zn XX (Na-like) satellites associated to the parent resonance lines. Again, an exhaustive identification of the spectral lines in the investigated wavelength range is as well presented, taking always into account the effect of plasma opacity on the observed spectra.
At the same time, each Zn XIX and Zn XX ion satellite line, caused by radiative transitions from highly excited autoionizing states of $\mathrm{Mg}$ - and $\mathrm{Na}$-like ions, comprises between six and about two dozens of nearly-equal-strength line transitions. So, a tabulation of the single components in such lines is practically unfeasible and a satisfactory simulation of these features is a computationally challenging task due to the enormous number of autoionizing levels that must be considered.

However, a complete list of the spectral features numbered in Figures 21 and 22 can be found in $[16,18]$, where a comparison between the calculated $\left(\lambda_{\text {calc }}\right)$ and experimentally measured $\left(\lambda_{\exp }\right)$ wavelengths of the spectral lines is reported. An excellent agreement is achieved between $\lambda_{\text {calc }}$ and $\lambda_{\text {exp }}$. Specifically, $\lambda_{\text {calc }}$ differs by less than $20 \mathrm{~m} \AA$ from $\lambda_{\exp }$ for most lines in all the charge states considered with a few differences above $50 \mathrm{~m} \AA$.

The spectra just showed in Figures 21 and 22 are a further evidence of the results already found in $[15,16]$ demonstrating that, in the case of a zinc target, many spectral lines produced by ns laser-plasma sources are Rydberg satellites associated to the Ne-like Zn XXI spectral features and especially represented by $\mathrm{Mg}$ - and $\mathrm{Na}$-like $\mathrm{Zn}$ ion lines.

Generally speaking, such satellites can be comparable or dominant relative to the intensity of the $\mathrm{Zn}$ XXI parent lines only in a plasma with $T_{e} \leq 300 \mathrm{eV}$. On the other hand, we have also observed spectral lines due to ions more highly charged than Zn XXI (e.g., O-like Zn XXIII or F-like Zn XXII ions), as shown for example in Figure 22.

This can be justified in terms of a spatial nonuniformity of the laser intensity on the focal plane, that can lead to hot laser spots responsible for the emission of the spectral lines due to the more highly charged F- and O-like $\mathrm{Zn}$ ions. In fact, taking into account that our data are spatially integrated across the face of the laser spot, we collect the contribution of these hot spots in the acquired spectra.

Furthermore, we generally work with time-integrated data over the whole experiment. As a consequence, it is impossible to establish if the observed spectral lines due to ions with high- and low-charge states are temporally coincident.

Beside all this, the time-integrated recorded spectra could not provide information about plasma time gradients. However, since in our case the laser pulse width is rather long (15 ns), a steady state regime is established during plasma heating. So, the integration over the time of the measured spectra is a drawback much less hard to overcome when compared to that met with radiative processes in plasmas produced by very short-heating-pulse (fs or ps) lasers. In addition, though our spectra are obtained without time resolution, it is reasonable to assume that only the most hot and dense plasma produced during laser illumination time is mainly responsible for the measured spectral lines, as confirmed by model calculations.

Identifications of the observed spectral lines (Figures 21 and 22) are obtained by comparison with the intensities of the collisional-radiative line transitions, properly calculated by the Hebrew University Lawrence Livermore Atomic Code (HULLAC) [67]. 
This package of codes is used to compute the atomic structure, transition rates, and wavelengths beside the synthetic line intensities for specific plasma parameters. The details of our application of HULLAC data in CR models have been previously presented [29]. In summary, the HULLAC suite of codes has been exploited to model the line emission from a zinc plasma.

More precisely, all level structure and rate coefficients have been calculated, all level energies and rate coefficients have been put into a CR rate matrix for the investigated ions, and the populations of the resulting steady state levels have been then evaluated. Moreover, the rates of radiative decay of all electric and magnetic dipole and quadrupole transitions (E1, M1, E2, and M2) are included in the modeling along with other prominent processes of plasma physics such as collisional excitation and deexcitation, collisional ionization, three body recombination, radiative recombination, excitation autoionization (EA), and radiationless capture leading to dielectronic recombination (DR) - that is, double electron transitions-especially responsible for the observed satellite lines (e.g., Mg- and Na-like $\mathrm{Zn}$ ion lines) along with multiple atom ionization.

Ion charge states from Mg-like Zn XIX to N-like Zn XXIV and the effects of plasma self-absorption (opacity) by means of escape factors have been also considered. At the same time, the assumptions of an isothermal, spherical zinc plasma with a diameter $L$ in the range (30-200) $\mu \mathrm{m}$-yet $30 \mu \mathrm{m}$ is a nonrealistic, lower limit-and of an ion temperature equal to electron one (i.e., $T_{i}=T_{e}$ ) have been included, as previously made for a barium plasma.

So, a comparison between simulated and measured spectra for a zinc plasma in a near Ne-like Zn XXI ion state is shown in Figures 23 and 24. The theoretical spectra have been worked out by taking the CR intensity for each line transition given by

$$
I_{j i}=n_{j} A_{j i}
$$

and spreading the intensity over a roughly normalized Voigt line profile $\phi(\nu)$, that is, [68],

$$
I(\nu)=\frac{1}{\sqrt{\pi}} I_{j i} \phi(\nu)
$$

Here, $A_{j i}$ denotes the total radiative transition rate between $j$ and $i$ levels, while $\phi(\nu)$, whose expression can be found in [18], includes Doppler, natural, and instrumental broadening terms.

The results of our model calculations have principally permitted to accurately reproduce the $\mathrm{Na}$ - and Mg-like Rydberg satellites excited by DR and recorded in the vicinity of Ne-like Zn XXI lines. These satellites are characterized by an emissivity $\varepsilon_{\mathrm{DR}}$ taking the form [18]

$$
\varepsilon_{\mathrm{DR}}=N_{e} N_{q+1} F_{1}\left(T_{e}\right) F_{2}(j, f),
$$

where $N_{e}$ is the free electron density, having a Maxwellian energy distribution, $N_{q+1}$ the density of the initial state of the recombining ion, $F_{1}\left(T_{e}\right)$ a function of the electron temperature and $F_{2}(j, f)$ the satellite-intensity factor owing to DR from level $i$ of ion $(q+1)$-via level $j$ of ion $q$ - to level $f$. Formally, $F_{1}\left(T_{e}\right)$ and $F_{2}(j, f)$ are, respectively, given by

$$
\begin{gathered}
F_{1}\left(T_{e}\right)=\frac{1}{2}\left(\frac{4 \pi a_{0}^{2} \mathrm{R}_{y}}{T_{e}}\right)^{3 / 2} e^{-\Delta E_{i j} / T_{e}}, \\
F_{2}(j, f)=\frac{g_{j}}{g_{i}} A_{j i}^{A I} \beta_{j f} .
\end{gathered}
$$

In these last two equations, $a_{0}$ is the Bohr radius, $\mathrm{R}_{y}$ the Rydberg unit of energy, $\Delta E_{i j}$ the energy difference between the initial level of the recombining ion (e.g., Ne- or Na-like) and a doubly excited level of the recombined ion (i.e., $\Delta E_{i j}$ is the capture energy of the free electron), $g_{j}$ and $g_{i}$ denote parameters previously introduced (11) for the intermediate (autoionizing) and initial (recombining) levels, $A_{j i}^{A I}$ is the autoionization rate from level $j$ to level $i$, and $\beta_{j f}$ is the radiative branching ratio from the upper level of the observed transition, related to $A_{j i}^{A I}$ and to total radiative transition rate $A_{j f}$ between $j$ and $f$ levels [18].

In Figure 23 a comparison between experimental (blue) and theoretical (pink, CR model and HULLAC package) soft $\mathrm{X}$-ray spectra for a zinc plasma in a near Ne-like $\mathrm{Zn}$ XXI ion state is reported within the wavelength range (8.3-8.7) $\AA$. Specifically, Figure 23 shows the $\mathrm{Mg}$ - and $\mathrm{Na}$-like satellites in the vicinity of the $n=4 \mathrm{Ne}$-like $\mathrm{Zn} \mathrm{XXI} \mathrm{resonance} \mathrm{lines} \mathrm{(4C,}$ $4 \mathrm{D}$ and $4 \mathrm{~F}$ ) for the same spectrum of Figure 21.

In this case, the modeling of the measured spectra is based on the following assumptions for the conditions of a spherical and isothermal zinc plasma produced by the ns Tor Vergata laser-plasma facility, similar to those already established for a barium plasma: (1) $T_{e}=250 \mathrm{eV}$, (2) $N_{e}=$ $10^{21} \mathrm{~cm}^{-3}$, (3) optical depths on all lines calculated for a $L=200 \mu \mathrm{m}$ plasma size, that is, the plasma opacity affects all observed spectral features, and (4) negligible fraction of hot electrons.

The good theory-experiment agreement is evident in the relative intensity of the $4 \mathrm{C}, 4 \mathrm{D}$ and $4 \mathrm{~F}$ spectral lines, so demonstrating the validity of the assumptions made for the ns TVLPS. Moreover, the satellite features observed in Figure 23 mainly are (a) the Na-like LMN (in what follows, a Zn XX or Zn XIX spectral feature takes a representation of the form $\mathrm{LM} n^{\prime \prime}$ or $\mathrm{LN} n^{\prime \prime}$, where the first character denotes a promotion of an $n=2$ electron, the second character indicates that the electron is migrated into the $n^{\prime}=3(\mathrm{M})$ or $n^{\prime}=4(\mathrm{~N})$ shell and, finally, the $n^{\prime \prime}$ character symbolizes the principal quantum number into which a free electron is captured $\left(n^{\prime \prime}=3, \ldots, 7\right.$ corresponding to letters from $M$ to $Q$, resp.)) and the $\mathrm{Mg}$-like LMN on the long wavelength side of the $4 \mathrm{D}$ resonance line; (b) the Na-like $\mathrm{LNN}$ between the $4 \mathrm{C}$ and $4 \mathrm{D}$ resonance lines.

Figure 24 shows, instead, a comparison between measured (blue) and calculated (pink, CR model, and HULLAC package) soft X-ray spectra for a zinc plasma in a near Nelike $\mathrm{Zn}$ XXI ion state in the spectral range (7.6-8.16) $\AA$. In particular, the $\mathrm{Mg}$ - and $\mathrm{Na}$-like satellites in the vicinity of the $n=5 \mathrm{Ne}$-like $\mathrm{Zn}$ XXI resonance lines (5C and 5D) are well visible in Figure 24 for the same spectrum of Figure 22 along with the two $4 \mathrm{~A}$ and $4 \mathrm{~B}(n=4)$ Ne-like $\mathrm{Zn}$ XXI resonance 
lines and with those due to F-like Zn XXII ions, properly emphasized in the figure and previously identified [16]. More in detail, the $4 \mathrm{~d} \rightarrow 2 \mathrm{p}$ transitions at $7.8865 \AA$ and $8.0435 \AA$ are well noticeable.

In these conditions, the experimental spectra have been simulated by making the same assumptions already adopted for reproducing the recorded spectrum of Figure 23 (i.e., $T_{e}=250 \mathrm{eV}, N_{e}=10^{21} \mathrm{~cm}^{-3}$, optically opaque plasma, no hot electrons) with the single difference that the plasma size is considered this time equal to $L=50 \mu \mathrm{m}$.

A reasonable agreement between theoretical and experimental results characterizes Figure 24, so again confirming the validity of the assumptions made for the ns TVLPS.

From this figure, it also evident that the $\mathrm{Na}$ - and $\mathrm{Mg}$ like LMO satellite features dominate the spectral structures near the parent $n=5 \mathrm{Ne}$-like Zn XXI lines. However, the higher- $n$ Mg-like LMP satellites are also observable on the long wavelength side of the 5D parent line, while the Nalike LMP and Mg-like LMQ satellite lines appear on the short wavelength side of the $5 \mathrm{C}$ resonance line.

Finally, the presence of satellite spectral structures on both the short and long wavelength sides of the Ne-like Zn XXI resonance lines is an evidence that the use of broadened line profiles for the diagnostics of hot and dense plasmas needs careful attention to the contribution coming from Rydberg satellite transitions, as shown in Figure 25, where the diagnostic utility of these lines can be foreseen.

More exactly, in Figure 25 the dependence of the Mg-, Na-, Ne-, F-, and O-like spectral structures on the plasma electron temperature $\left(T_{e}\right)$ within the soft X-ray wavelength ranges (7.6-8.2) $\AA$ (panel (a)) and (8.3-8.9) $\AA$ (panel (b)) is theoretically simulated. As a result, models for the Mg- and Na-like Rydberg satellite lines have been properly developed for $n \leq 5$, especially for $n=4$ and $n=5$.

Figure 25 also shows that the satellites due to Na-like $\mathrm{Zn} \mathrm{XX}$ ions are powerful up to electron temperatures of $T_{e}=300 \mathrm{eV}$, while the O-like and F-like spectral lines are prevailing for $T_{e}>300 \mathrm{eV}$, as for example observed in the panel (a) for the 2-4 O-like and 2-5 F-like transitions falling into the range (7.2-8.2) $\AA[16]$.

At the same time, the O-like $2 s \rightarrow 3 p$ transitions are observable in the spectra calculated at the highest electron temperatures reported in the panel (b) of Figure 25. In addition, the Ne-like Zn XXI resonance lines are optically thick for $T_{e} \leq 400 \mathrm{eV}$. In particular, opacity effects can be observed on the $4 \mathrm{~F}$ and $4 \mathrm{G}$ spectral features. Considering higher values of $T_{e}$, the fraction of Ne-like $\mathrm{Zn} \mathrm{XXI} \mathrm{ions} \mathrm{in} \mathrm{the}$ plasma is too low, and the opacity effects on the resonance lines are not more so strong.

On the other hand, theoretical simulations also demonstrate that the Na-like Zn XX satellites are optically thin for an electron density $N_{e} \leq 10^{21} \mathrm{~cm}^{-3}$ and that satellite intensity depends on plasma temperature. So, a dependence of the satellite features on the plasma parameters is ascertained.

In summary, such considerations permit to establish that, if the plasma ions emitting the satellite spectral structures are spatially placed near to the laser-matter-interaction region, the Rydberg satellites can be effectively used as a local diagnostic tool for a reliable evaluation of plasma

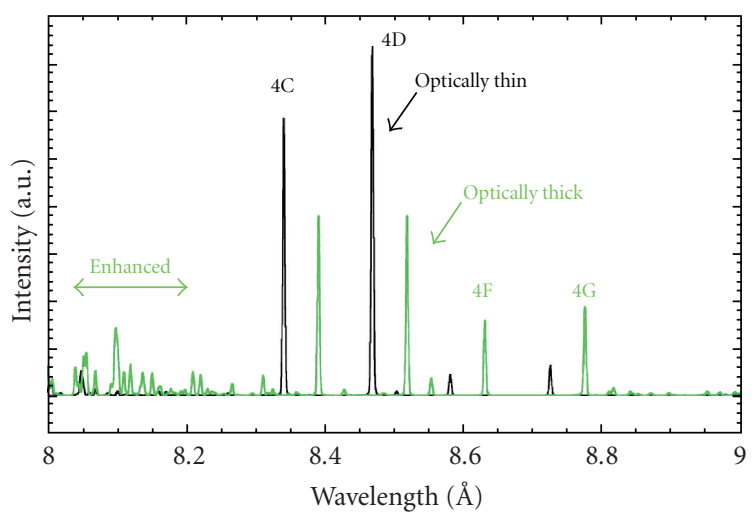

Figure 26: Soft X-ray spectra due to O-, F-, and Ne-like Zn ions computed in the range (8-9) $\AA$ for an optically thin and thick plasma. Note that the spectrum with opacity effects (optically thick) has been offset by $50 \mathrm{~m} \AA$.

conditions, as foreseen in [69]. Moreover, the presented spectral data, especially the $n=4$ and $n=5 \mathrm{Ne}$-like Zn XXI resonance lines, are very optically thick, that is-generally speaking - the $n \mathrm{C}$ and $n \mathrm{D}$ spectral features are influenced by large optical depths, strictly related to plasma sizes (10), so confirming what previously observed for the emission spectra of a barium plasma.

The opacity effects [29] of the studied plasmas are well noticeable and can be summarized in four main points: (1) the plasma average charge state is driven higher by enhanced ionization from more-highly-populated Zn XXI upper levels, (2) enhancing spectral lines due to higher ion charge states such as those F-like and O-like, (3) relative intensity of the weaker Ne-like Zn XXI lines is increased by collisionally coupling the upper states of the $4 \mathrm{C}$ and $4 \mathrm{D}$ lines to those of the $4 \mathrm{~F}$ and $4 \mathrm{G}$ spectral features and (4) enlargement of the spectral lines.

A visual evidence of some opacity effects on the plasma emission spectra is illustrated in Figure 26.

Specifically, in Figure 26 the simulated emission spectra due to $\mathrm{O}-,, \mathrm{F}-$, and Ne-like $\mathrm{Zn}$ ions in the vicinity of the $n=4 \mathrm{Zn}$ XXI resonance lines, calculated in the soft X-ray spectral range (8-9) $\AA$ with and without opacity effects (i.e., for optically thick and thin plasma, resp.), are reported.

These synthetic spectra have been obtained in a similar way to that previously described by assuming a spherical plasma with uniform density and temperature taking the values $N_{e}=10^{21} \mathrm{~cm}^{-3}$ and $T_{e}=T_{i}=300 \mathrm{eV}$, respectively, by self-consistently computing the ion level populations and by properly considering escape factors.

Finally, the growing of the intensity of the F-like, the $4 \mathrm{~F}$ and the $4 \mathrm{G}$ spectral features is clearly visible in Figure 26 when opacity effects (i.e., escape factors) are entered in the calculations.

As last example of spectroscopic inspection, in Figure 27 we report soft X-ray emission spectra recorded with high spectral (typically $\lambda / \Delta \lambda \cong 4000)$ and spatial $(20-50 \mu \mathrm{m})$ resolution in the wavelength range (14-16) $\AA$ for an iron plasma generated by the ns Tor Vergata laser-plasma facility working at three different conditions: (a) $6 \mathrm{~J}$ laser energy and 


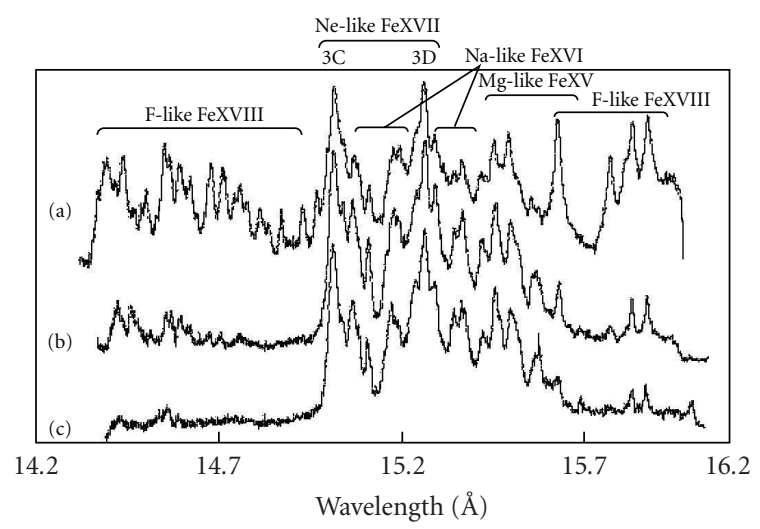

FIGURE 27: Soft X-ray emission spectra measured in the wavelength range (14-16) $\AA$ for a dense and hot iron plasma produced on a $60 \mu \mathrm{m}$ thick $99.9 \%$ pure Fe foil by means of the ns TVLPS operating at three different conditions: (a) $6 \mathrm{~J}$ laser pulse energy, $200 \mu \mathrm{m}$ laser spot size, (b) $4 \mathrm{~J}$ laser pulse energy, $500 \mu \mathrm{m}$ laser spot size, and (c) $2 \mathrm{~J}$ laser pulse energy, $500 \mu \mathrm{m}$ laser spot size.

$200 \mu \mathrm{m}$ laser spot size, (b) $4 \mathrm{~J}$ laser energy and $500 \mu \mathrm{m}$ laser spot size, and (c) $2 \mathrm{~J}$ laser energy and $500 \mu \mathrm{m}$ laser spot size [19].

The various working conditions of the Nd:YAG/Glass laser source have permitted to attain a varying pulse intensity on the focal plane in an attempt to give rise to different plasma states strictly related to laser intensity. In fact, the plasma electron temperature can be decreased/increased by reducing/growing the laser energy or by defocusing/focusing the laser beam on the target opportunely moving the lens with respect to the best focus position, that is, by decreasing/increasing the laser intensity on the focal plane.

As a result, diverse iron spectral structures can be observed for different values of laser intensity illuminating the target. Specifically, Figure 27 shows the presence of the dielectronic Mg-like Fe XV and Na-like Fe XVI satellite lines in the vicinity of the two Ne-like Fe XVII $3 d \rightarrow 2 p 3 C$ and $3 \mathrm{D}$ resonance lines at $15.014 \AA$ and $15.267 \AA$, corresponding to the strong $2 p^{5} 3 d \rightarrow 2 p^{6}\left({ }^{1} P_{1} \rightarrow{ }^{1} S_{0}\right)$ and $2 p^{5} 3 \mathrm{~d} \rightarrow 2 \mathrm{p}^{6}$ $\left.\left({ }^{3} \mathrm{P}_{1} \rightarrow{ }^{1} \mathrm{~S}_{0}\right)\right)$ transitions, respectively. These resonance lines have been recorded with a lower spectral resolution of the order of $\lambda / \Delta \lambda=1200-1500$ than the surrounding satellite lines because of the remarkable optical absorption of the plasma.

The spectral features of the more highly charged F-like Fe XVIII ions are also well visible in Figure 27-especially in the spectrum (a) - though a reduction of laser intensity (i.e., plasma temperature) is responsible for a partial or quasitotal suppression of the X-ray emission from the higher charged (e.g., O- and F-like) iron ions compared to that from the Naand Mg-like ions with a lower charge state (spectra (b) and (c)).

An accurate identification of the observed spectral lines can be found in [19]. This classification has been obtained by comparisons with the HULLAC modeling and previous Brown et al. results [70, 71] focused on the spectral analysis in the low density plasmas with a less spectral resolution $(\lambda / \Delta \lambda \cong 500)$ than that characterizing our measurements.
Specifically, the higher spectral resolving power of our results enables one to split several lines not resolved in Brown works and to record detailed spectra of the closely spaced satellite lines emitted by $\mathrm{Na}$ - and $\mathrm{Mg}$-like Fe ions.

In summary, the comparisons with the HULLAC calculations and Brown measurements have permitted to identify the O-, F-, and Ne-like Fe ion lines, while the satellite spectral structures from $\mathrm{Na}$ - and $\mathrm{Mg}$-like $\mathrm{Fe}$ ions have been classified through comparisons only with the HULLAC calculations.

In particular, the HULLAC package of codes has been used to compute the theoretical spectra at $T_{e}=200 \mathrm{eV}$ and $N_{e}=10^{21} \mathrm{~cm}^{-3}$, that is, for plasma conditions similar to those previously found.

Within this framework, a faithful modeling of the measured spectral lines-especially for the $\mathrm{Ne}-$, $\mathrm{Na}-$, and Mg-like iron features-has been obtained when both the dielectronic recombination and the excitation autoionization processes have been included along with the opacity effects for plasma dimensions of $200 \mu \mathrm{m}$. At the same time, a few experimental intensities of the stronger lines emitted by Flike Fe ions have been adequately reproduced by HULLAC though many of the F-like spectral structures recorded in the range (14-15) $\AA$ were characterized by a much stronger intensity compared to that simulated by the HULLAC model.

Anyhow, a good level of accuracy between theoretical and experimental spectra has been achieved, thus permitting to associate an observed line to an expected atomic transition. More precisely, the majority of the calculated HULLAC spectral lines differs by less than $20 \mathrm{~m} \AA$ from the measured ones with a few differences above $50 \mathrm{~m} \AA$. On the other hand, our measurements are also in excellent agreement with those of Brown since the difference between our and Brown results is less than $8 \mathrm{~m} \AA$ for most of the spectral lines.

In addition, the spectral identification has been easy for most of the O-, F-, and Ne-like Fe lines, while it is resulted more intricate for the Na- and Mg-like satellite features. In fact, though a high-resolution spectroscopy has been used, many of these lines have been difficult to recognize because of line blending.

Such a investigation has been developed by taking into account that the evaluation of the main features of hightemperature and high-density plasmas can be a noticeable study topic in the astrophysics field, where these information, inferring by the analysis of X-ray spectra and especially by the investigation of spectral line intensity of highly charged ion states such as those of iron, are fundamental for a modeling of the star parameters in an attempt to reproduce the conditions charactering the various stellar shells.

Finally, the experiments concerning the acquisition of iron plasma spectra have been performed as before for barium and zinc plasmas keeping constant the laser conditions for each acquired spectrum with the laser energy varying less than $\pm 5 \%$. The spectral features have been obtained by shooting 1-10 laser shots on a stepped rotating target (step height varying in the range $300-800 \mu \mathrm{m}$ ), composed by a $60 \mu \mathrm{m}$ thick $99.9 \%$ pure Fe tape glued to the surface of the higher Teflon step.

The iron and Teflon targets have been translated into the laser beam on different shots in order to record Fe and 


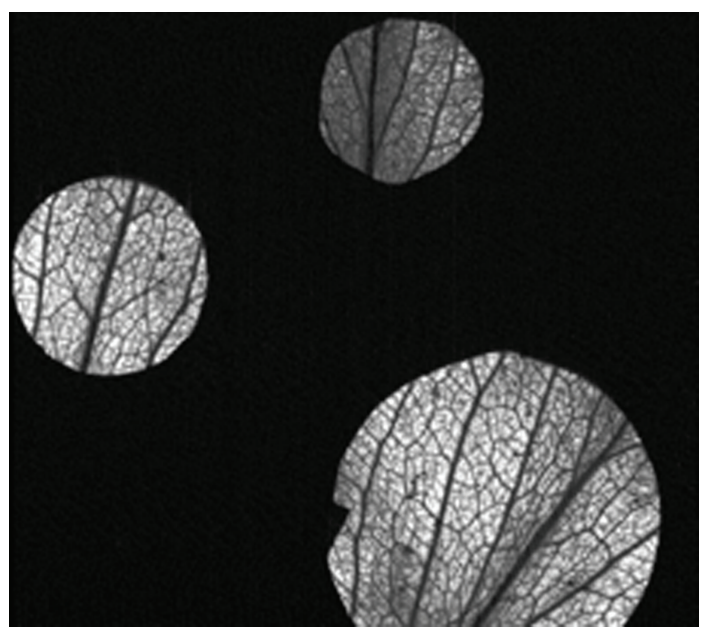

FIGURE 28: Digital microradiography of withered eucalyptus leaves obtained by the TVLPS using a $\mathrm{Cu}$ rotating tape target. The radiographic image has been acquired with laser energy of $9 \mathrm{~J}, \mathrm{CCD}$ exposure time and operating temperature of $1 \mathrm{~s}$ and $-20^{\circ} \mathrm{C}$, respectively, and a $5 \mathrm{keV}$ maximum energy of the emitted soft X-rays.

Teflon (i.e., fluorine) spectra on the same film, physically separated thanks to the step on each target. In particular, the Teflon plasma has been used for producing the $\mathrm{H}$ like and He-like reference spectral lines for the wavelength calibration of the various spherically bent spectrometers employed in the experiment. Finally, taking into account the dispersion curves of the spectrometers, a total uncertainty less than $10 \mathrm{~m} \AA$ is introduced in the absolute wavelength measurements (typically the error is $1-2 \mathrm{~m} \AA$ ).

\section{Applications: X-Ray Imaging of Thin Biological Samples}

A further and important application of soft X-rays emitted by laser-induced plasma is represented by imaging (i.e., microradiographies) of thin biological samples [4, 21, 23]. This application, based on the radiation selective uptake, can be used in the field of radiobiology for visualizing specific structures and detecting bioaccumulation sites due to specimen contamination from pollutants (e.g., heavy metals).

A first digital microradiography of withered eucalyptus leaves has been recorded by the CCD camera previously described (Section 2.3) and it is shown in Figure 28.

From Figure 28, it is worth to notice the high quality of the obtained radiographic image, characterized by a high spatial resolution and a high contrast. Specifically, the different absorption of soft X-rays emitted by the TVLPS in the various areas of the sample permits to emphasize the leaf structures, with main and minor veins clearly visible.

The radiographic digital image has been obtained by locating in this order the following elements on a copper support with holes having diameters in the range (1-5) $\mathrm{mm}$ : an aluminium filter with a thickness of $15 \mu \mathrm{m}$, the eucalyptus leaves, and a $7 \mu \mathrm{m}$ Kapton filter close to CCD chip (less than $1 \mathrm{~cm})$.
The two filters have been used for different purposes. More precisely, Al foil permits to stop visible and UV radiation emitted by plasma, while Kapton layer efficiently protects CCD from debris particles coming from copper solid target released during laser-plasma generation.

Moreover, a thin copper rotating tape target with a thickness of $50.8 \mu \mathrm{m}$ has been illuminated by GW-level Nd:YAG/Glass laser pulses with an energy of $9 \mathrm{~J}$, properly focused by a triplet lens at $-45^{\circ}$ incidence angle relative to the target normal.

The CCD camera, cooled for attaining a working temperature of $-20^{\circ} \mathrm{C}$, has been located inside the vacuum chamber at a distance of $15 \mathrm{~cm}$ from the target point where laserinduced plasma is generated along an angle of about $+45^{\circ}$ with respect to the target normal (Figure 1). A CCD exposure time of $1 \mathrm{~s}$ has been needed for recording the radiographic digital image.

Finally, Quantrad PIN photodiode with its active surface shielded by a $40 \mu \mathrm{m}$ aluminum layer for stopping visible and UV components of plasma radiation has been placed at circa $90^{\circ}$ relative to the target normal.

As previously mentioned, microradiographies of biological samples are useful for the mapping of the bioaccumulation sites due to contamination by heavy metals (e.g., cadmium) and pollutants. For this purpose, Viola tricolor leaves with various doping concentrations have been properly prepared in the controlled laboratory conditions.

These specimens have been exposed to the same experimental working parameters as the eucalyptus leaves.

The radiographic images, always recorded by CCD camera with an exposition time of $1 \mathrm{~s}$ and an operating temperature of $-50^{\circ} \mathrm{C}$, show various granule structures of Viola tricolor leaves (Figure 29), especially observed where soft X-rays emitted by laser induced plasma are more strongly absorbed.

Specifically, as a validation of the results obtained using films as recording media, these leaf structures with a high X-ray absorption, corresponding to darker regions of the microradiographies, are more evident for higher doping levels [72].

So, the contrast between bioaccumulation sites and sample background can be well highlighted by means of both qualitative visual analyses and quantitative surveys of line profiles along the granule structures by using suitable image processing software.

Preliminary results concerning bioaccumulation analysis are qualitatively shown in Figure 29 for Viola tricolor leaves exposed to $\mathrm{CuSO}_{4}(2 \%)$ at three pollution levels: control (no pollution), contamination for 4 hours, and contamination for 24 hours.

The outcomes are based on qualitative visual studies of the obtained radiographic images. They show that the biological samples with a higher contamination level are darker than the others (especially the control) due to a stronger absorption of soft X-rays.

This result establishes an evidence of a higher concentration of pollutants in the sample areas characterized by a remarkable X-ray uptake. 


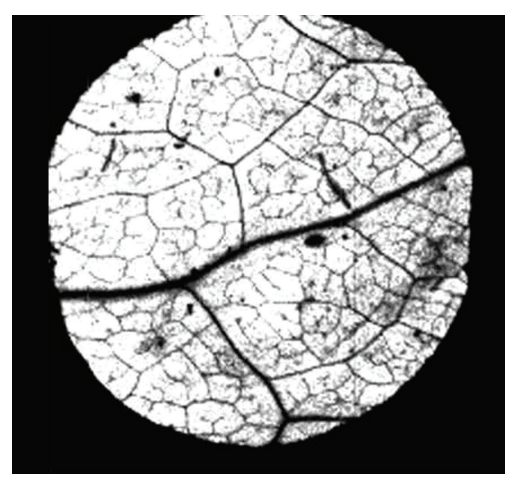

(a)

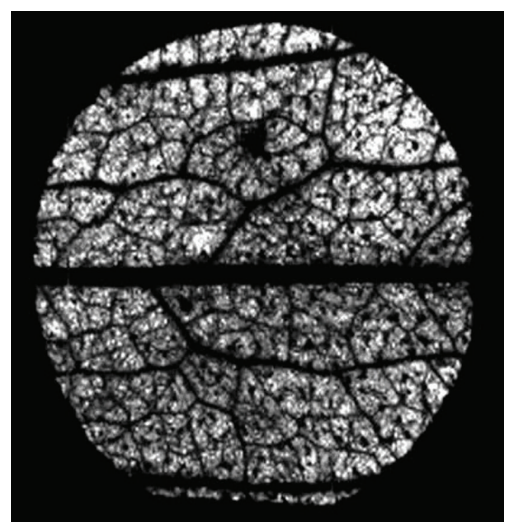

(d)

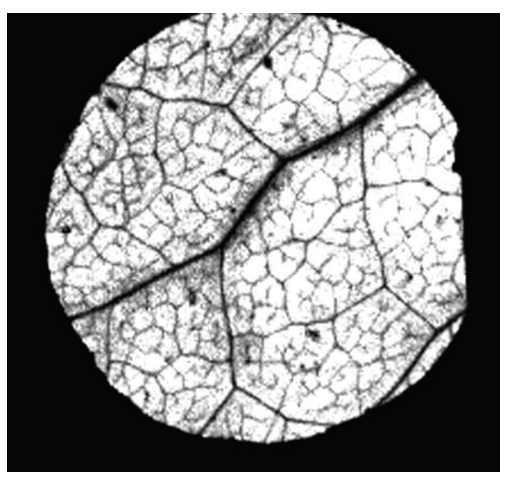

(b)

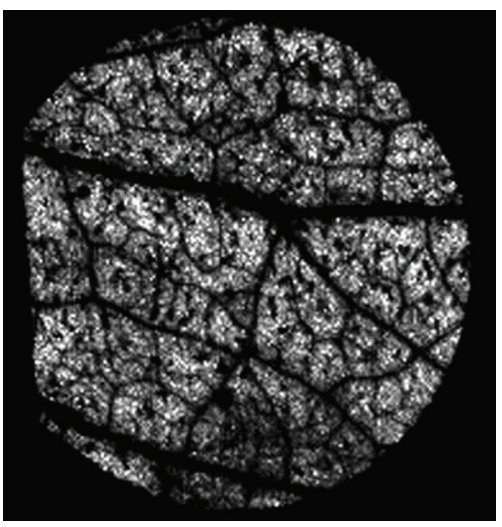

(e)

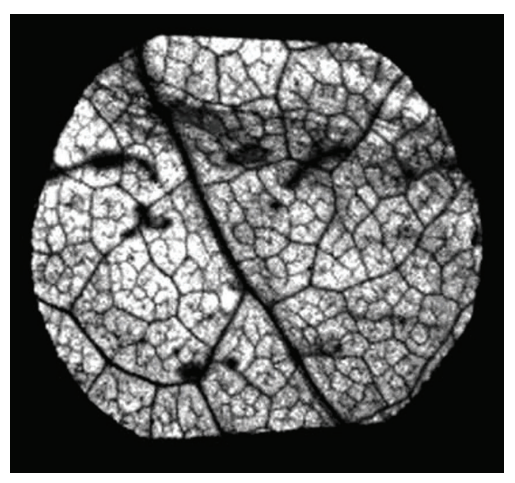

(c)

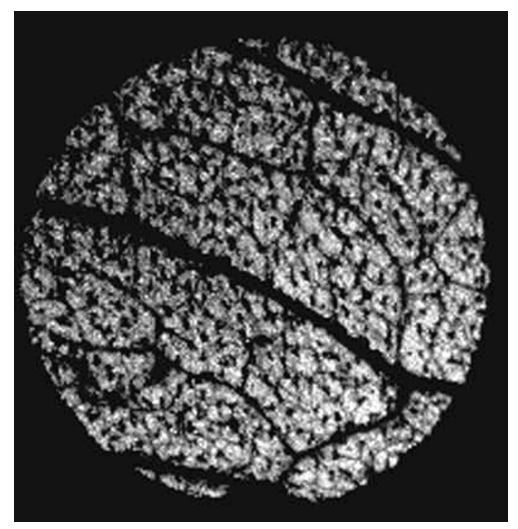

(f)

FIgURE 29: Digital microradiographies of Viola tricolor leaves with increasing doping: control (no contamination, two top left images), doping for 24 hours (two bottom right images), doping for 4 hours the remaining. The radiographic images, recorded by CCD camera ( $1 \mathrm{~s}$ exposure time, $-50^{\circ} \mathrm{C}$ cooling temperature) with a $5 \mathrm{keV}$ maximum energy of the emitted soft X-rays, have been obtained by the TVLPS illuminating a $\mathrm{Cu}$ rotating tape target with a laser energy of $9 \mathrm{~J}$. A different darkness due to a diverse X-ray absorption is clearly visible, and it is an evidence of the various doping levels on Viola tricolor leaves.

\section{Conclusions}

In this paper, a wide review of some main results obtained in the field of plasma physics by using the ns TVLPS has been presented. More precisely, the Tor Vergata laserplasma facility used in the experiments, based on a GWlevel, multistage, pulsed, tabletop Nd:YAG/Glass laser (pulse width $=15 \mathrm{~ns}$ ), has been properly described along with the devices (i.e., nonstandard, home-assembled CCD camera and spherical crystal-based spectrometer) employed for soft $\mathrm{X}$-ray imaging (microradiographies) and spectroscopy.

During the experiments the tabletop Nd:YAG/Glass laser system has worked with a pulse energy varying in the range (1-10) J and with a maximum peak intensity of about $10^{13} \mathrm{~W} / \mathrm{cm}^{2}$.

A few principal applications of laser induced plasmas were discussed, especially in the following fields: (1) conversion efficiency $\eta$ from IR laser radiation to soft X-photons in the spectral region (1.3-1.55) keV, corresponding to (89.56) $\AA$ wavelength range, (2) spatially resolved soft X-ray emission spectroscopy with high spectral $(\lambda / \Delta \lambda$ theoretically varying within the range 3000-10000) and high spatial (usually varying between $20 \mu \mathrm{m}$ and $50 \mu \mathrm{m}$ ) resolution employing stepped rotating solid targets and various spherically bent spectrometers, and (3) soft X-ray imaging, especially in the field of microradiographies of thin biological samples, by means of an astronomical grade, front illuminated, highsensitivity, high-resolution (about $15 \mu \mathrm{m}$, corresponding to pixel size) CCD camera.

The use of stepped rotating targets, composed by two parts (i.e., the investigated target and the reference one) and opportunely translated into the laser beam on different shots, is fundamental for recording two well-separated spectra on the same film concerning the considered target (e.g., $\mathrm{Ba}, \mathrm{Zn}$, and $\mathrm{Fe}$ ) and that taken as a reference (e.g., $\mathrm{Mg}$ and $\mathrm{Al}$ ) for the recorded spectral lines, respectively.

On the other hand, the use of more spectrometers based on mica or quartz spherical crystals, for covering the studied wavelength range, permits to achieve a much higher instrumental spectral resolution (typically $\lambda / \Delta \lambda \cong 4000$ ) 
than that possible with a single spherically bent spectrometer.

However, the effective spectral resolving power for such spectrometers can be less than 4000 , since it is essentially limited by the Doppler broadening of the spectral lines due to plasma expansion.

Concerning the aforesaid point (1), the IR $\rightarrow$ soft Xray conversion efficiency has been surveyed for six different solid targets (magnesium, titanium, iron, copper, zinc, and yttrium). This study has mainly showed that the conversion efficiency: (1.1) depends on the type of target (i.e., on the atomic number $Z$ ) since it is related to the resonance line position-for example, within the spectral range (1.31.55) $\mathrm{keV}$, lines 5C, 5D and $4 \mathrm{C}, 4 \mathrm{D}$ for $\mathrm{Cu}$ and $\mathrm{Zn}$, respectively (see $[13,18,29]$ and Figure 21) —of the levels involved in the X-ray emission, (1.2) changes by varying the laser intensity on the target, that is specifically increases/decreases by increasing/decreasing the laser pulse intensity on the focal plane according to a quasi-linear saturating trend, (1.3) depends on the considered soft X-ray spectral region, and (1.4) in the investigated spectral range (i.e., $1.3-1.55 \mathrm{keV}$ ) and for the analyzed targets assumes the maximum values for copper $(Z=29)$ and zinc $(Z=30)$, with almost the same mean value $\langle\eta\rangle \cong 0.22 \%$.

Moreover, still for point (1), it is possible to assert that IR $\rightarrow$ soft X-ray conversion efficiency is less than $1 \%$ within the considered spectral range. The remaining fraction of laser energy illuminating the target not reflected by plasma (nearly $80 \%$ in our case) is converted into visible and ultraviolet radiation emitted by plasma and, especially, transformed into the heat necessary to increase the target temperature from $25^{\circ} \mathrm{C}$ to about $10^{6 \circ} \mathrm{C}$ (plasma temperature in our case).

Concerning the aforesaid point (2), we presented some spectroscopic results obtained by using the soft $\mathrm{X}$-rays emitted by our laser-plasma facility. A useful discussion of the observed spectra was also undertaken for three different solid targets (barium, zinc and iron) on which the laser beam is focused for plasma generation.

Specifically, comparisons between CR model calculations simulating the emission by nanosecond laser induced plasma and high spectrally resolved measurements were well investigated.

Within this framework, the merging between experimental results on high-resolution spectra and nanosecond laser pulses, neglecting the influence of hot electrons, permitted a more precise identification of the plasma ions responsible for the spectral emission and a better comparison between theoretical and experimental spectra.

In particular, measurements and modeling of the emission of a barium plasma in the soft X-ray spectral range (7.8-9.5) $\AA$ for high- $n$ Rydberg levels in a near Ni-like ionization state were analyzed. In this case, the ATOMIC code calculations were used for evaluating the transitions between levels involved in X-ray emission. This modeling is reasonably accurate and permits line identifications in observed spectra, especially if the MUTA calculations are included and compared with a detailed level-to-level approach including configuration interaction.
A similar study was also presented for a zinc plasma with specific reference to the measures and identifications of the $L$-shell Rydberg series in the near Ne-like Zn XXI spectrum (wavelength range 7.6-8.7 $\AA$ ) with a major focusing on the high- $n$ dielectronic Rydberg satellites from Mg-like Zn XIX and Na-like $\mathrm{Zn} \mathrm{XX}$ ions. In fact, the obtained spectra are characterized by spectral features that arise from enormous manifolds of dielectronic-recombination-fed satellites in the vicinity of the Ne-like $\mathrm{Zn}$ XXI parent resonance lines.

Moreover, it was observed that these satellites converge to the red edge of the parent line, especially for the $n=4 \mathrm{Zn}$ XXI lines. So, in simulations of Stark broadened line profiles for high- $n$ transitions of the Ne-like Zn XXI $n \mathrm{C}$ and $n \mathrm{D}$ series, the large contribution given by these satellites to the broadened line shape has to be considered if one wishes to obtain accurate diagnostic information.

CR model calculations were allowed to deduce that the dielectronic Rydberg satellites have a remarkable weight in zinc plasmas with $T_{e} \leq 300 \mathrm{eV}$, while for higher- $Z$ elements the Rydberg satellites provide an important contribute to the spectra only in plasmas with significantly higher temperatures.

At the same time, optically thick lines characterize our $\mathrm{Zn}$ plasma emission spectra. This is especially true for $n=$ 4 and $n=5 \mathrm{Ne}$-like Zn XXI spectral features that show the typical behavior of the lines observed in plasmas where opacity effects must be considered. As a consequence, a direct inspection of plasma conditions near the laser-matterinteraction region is complicated by the complexity of the transport modeling of these lines.

On the contrary, the Na-like Zn XX satellite features were observed to be optically thin. Thus, considering spatially resolved, high-resolution measurements, the Rydberg satellites can be used as a local diagnostic tool of plasma conditions in the laser-target-interaction region though a complete investigation of such spatially resolved observations is necessary for $L$-shell emitting ions.

Finally, the spectroscopic investigation by highresolution emission spectra recorded in spectral range between $14 \AA$ and $16 \AA$ for an iron plasma produced by the TVLPS enabled one to observe various spectral structures, such as dielectronic Mg-like Fe XV and Na-like Fe XVI satellite lines, two Ne-like Fe XVII 3C and 3D parent resonance lines at $15.014 \AA$ and $15.267 \AA$, respectively, and Flike Fe XVIII spectral features. Such spectral structures, that can be exploited for studies in the astrophysical field, were measured for different values of laser intensity illuminating the target and 3C and 3D lines are blended with the Na-like and $\mathrm{Mg}$-like satellites.

In the specific cases of zinc and iron plasmas, model calculations to reproduce experimental spectra were performed by using the HULLAC suite of codes. More exactly, the line identifications in the observed spectra were attained by comparisons with CR transition intensities computed by HULLAC taking into account the dielectronic recombination and excitation autoionization phenomena.

In all analyzed cases, the agreement between calculated and measured spectra is good though some recorded spectral features are not well reproduced having a different intensity 
compared to that obtained by simulations. Moreover, for most of the lines, the theoretical wavelengths differ by less than $20 \mathrm{~m} \AA$ from the measured ones with a few gaps above $50 \mathrm{~m} \AA$.

This raises the possibility of exploiting the proposed modeling for highly charged ionization stages in order to obtain useful diagnostic tools, such as the sensitivity of line intensities/ratios to plasma temperature/density, in an attempt to simulate plasma conditions (e.g., electron temperature and density, opacity, and fraction of hot electrons).

The considered studies have also shown that the satellites features have an important role in the field of plasma diagnostics and that the plasma conditions are strongly depending on laser intensity.

In fact, the strength of the spectral lines from higher charged ions (e.g., O- and F-like lines in the case of iron plasma spectrum) increases for growing laser intensity, while a partial or total suppression of these lines is observed for decreasing values of laser intensity. In this last situation, the dominant spectral features are those from lower charged ions (e.g., $\mathrm{Na}$ - and $\mathrm{Mg}$-like lines for iron plasma spectrum). This occurs because the plasma temperature and, consequently, the various fractions of the different ionization states are a growing function of laser intensity.

By exploiting the proposed modeling of the measured soft X-ray spectra, a good estimation of plasma parameters was also made for the TVLPS. Specifically, by a comparison between theoretical simulations and measured spectra, we evaluated that, in the case of our experiments, the conditions of the generated plasma were characterized by the following main parameters in the assumption of isothermal and spherical plasma:

(2.1) $200 \mathrm{eV} \leq T_{e} \leq 300 \mathrm{eV}$ (electron temperature),

(2.2) $T_{i}=T_{e}$,

(2.3) $10^{20} \mathrm{~cm}^{-3}<N_{e} \leq 10^{21} \mathrm{~cm}^{-3}$ (electron density),

(2.4) opacity, related to plasma dimensions typically ranging within (50-300) $\mu \mathrm{m}$,

(2.5) negligible fraction of hot electrons.

Considering electron density values (point 2.3) compared to critical density and plasma reflectivity studies [33], it is also possible to conclude that the laser radiation absorbed by plasma is about $80 \%$ whose less than $1 \%$ is converted into $\mathrm{X}$-ray radiation.

However, the nonperfect agreement between theoretical and experimental spectra can be considered as an indication that significant gradients of temperature and density may exist in the plasma, whose effects are observable in the recorded spectra.

Finally, a further application of the TVLPS in the field of $\mathrm{X}$-ray imaging was reported (point (3)).

Particularly, preliminary high-quality, high-resolution, digital microradiographies of thin biological samples, exploiting soft $\mathrm{X}$-rays with a $5 \mathrm{keV}$ maximum energy, were obtained by directly recording radiographic images on a thick, front illuminated, astronomical grade, nonstandard, home-assembled, cooled (up to $-80^{\circ} \mathrm{C}$ ) CCD camera with a spatial resolution of $2 k \times 2 k$ pixels and a pixel size of $15 \mu \mathrm{m}$. This CCD camera is also characterized by good cosmetic and photometric quality and it is optimized for very low flux imaging and spectroscopy in visible and $\mathrm{X}$-ray regions.

The microradiographies were recorded with a $1 \mathrm{~s}$ CCD exposition time and an operating temperature of $-20^{\circ} \mathrm{C}$ and $-50^{\circ} \mathrm{C}$. The acquired radiographic images permitted to recognize the main structures of the sample (e.g., main and minor veins, granule compositions, etc.) and the possible presence of contaminant substances (e.g., heavy metals) on the surface by exploiting different absorption of soft X-rays in the various areas of the investigated sample.

In fact, sample regions with a higher X-ray absorption correspond to darker regions of the microradiographies where higher doping levels are achieved. So, the contrast between bioaccumulation sites and sample background can be well emphasized by using suitable image processing software that permit both the qualitative visual analyses and the quantitative investigations by using line profiles along the sample structures. Hence, an accurate study of the distribution of pollutant bioaccumulation sites on the sample surface can be undertaken.

As future perspective, in order to extend the spectroscopic investigations and the analysis of biological samples at higher energy (e.g., $10 \mathrm{keV}$ ), we are scheduling to increase the laser energy by adding a fifth amplification stage to the TVLPS.

In this manner, it will be possible to increase the energy of laser beam above the current maximum value of $10 \mathrm{~J}$ and to raise the laser intensity on the target above the present highest value of $10^{13} \mathrm{~W} / \mathrm{cm}^{2}$ for enhancing X-ray generation. The scheduled increasing of the laser energy above $10 \mathrm{~J}$ might be ultimately useful for finding a definitive validation of the saturation effect observed in the IR $\rightarrow$ soft X-ray conversion efficiency, that is a reliable law and a justification for the trend of the conversion efficiency against laser energy.

Moreover, we are also scheduling to take in the next future pinhole camera pictures on a plasma scale-length using a CCD camera or the $1 \mathrm{D}$ imaging by spherically bent spectrometer.

\section{Acknowledgments}

This is a review paper on principal results obtained at the Quantum Electronics and Plasma Laboratory of the Tor Vergata University (Rome) in collaboration with several worldwide research groups. The authors wish to thank all the scientists that have collaborated at the achievement of the presented results and, especially, at the theoretical analysis and modeling. A special acknowledgment for the given contribution is addressed to Drs. A. Y. Faenov and T. A. Pikuz.

\section{References}

[1] R. Goldston and P. H. Rutherford, Introduction to Plasma Physics, Institute of Physics Publishing, Philadelphia, Pa, USA, 1995.

[2] M. D. Perry and G. Mourou, "Terawatt to petawatt subpicosecond lasers," Science, vol. 264, no. 5161, pp. 917-924, 1994. 
[3] G. A. Mourou, C. P. J. Barty, and M. D. Perry, "Ultrahighintensity lasers: Physics of the extreme on a tabletop," Physics Today, vol. 51, no. 1, pp. 22-28, 1998.

[4] S. Martellucci, M. Francucci, and P. Ciuffa, "Ultraintense tabletop laser system and plasma applications," in Progress in Ultrafast Intense Laser Science (PUILS), vol. I of Springer Series in Chemical Physics, Springer, Berlin, Germany, 2006.

[5] S. Martellucci, C. Bellecci, M. Francucci et al., "Soft x-ray generation by a tabletop Nd:YAG/glass laser system," Journal of Physics Condensed Matter, vol. 18, no. 33, pp. S2039-S2044, 2006.

[6] W. L. Kruer, Physics of Laser Plasma Interaction, Addison Wesley, Redwood City, Calif, USA, 1988.

[7] I. C. E. Turcu and J. B. Dance, $X$ rays from laser plasma: Generation and Applications, Wiley \& Sons, London, UK, 1998.

[8] E. P. Liang, S. C. Wilks, and M. Tabak, "Pair production by ultraintense lasers," Physical Review Letters, vol. 81, no. 22, pp. 4887-4890, 1998.

[9] J. Lindl, "Development of the indirect-drive approach to inertial confinement fusion and the target physics basis for ignition and gain," Physics of Plasmas, vol. 2, no. 11, pp. 39334024, 1995.

[10] W. J. Hogan, R. Bangerter, and G. L. Kulcinski, "Energy from inertial fusion," Physics Today, vol. 45, no. 9, pp. 42-50, 1992.

[11] B. A. Remington, D. Arnett, R. P. Drake, and H. Takabe, "Modelling astrophysical phenomena in the laboratory with intense lasers," Science, vol. 284, no. 5419, pp. 1488-1493, 1999.

[12] B. A. Remington, R. P. Drake, H. Takabe, and D. Arnett, "A review of astrophysics experiments on intense lasers," Physics of Plasmas, vol. 7, no. 5, pp. 1641-1652, 2000.

[13] K. B. Fournier, A. Y. Faenov, T. A. Pikuz et al., "Observations of high-n transitions in the spectra of near-neon-like copper ions from laser-produced plasmas," Journal of Physics B, vol. 35, no. 15, pp. 3347-3364, 2002.

[14] I. Y. Skobelev, A. Y. Faenov, T. A. Pikuz et al., "Spectral transitions from the rydberg autoionization states of a Li-like $\mathrm{Mg}$ X ion," Journal of Experimental and Theoretical Physics, vol. 95, no. 3, pp. 421-428, 2002.

[15] K. B. Fournier, A. Y. Faenov, T. A. Pikuz et al., "Rydberg transitions in the spectra of near-neon-like $\mathrm{Cu}$ and $\mathrm{Zn}$ ions in different laser-produced plasmas: Observations and modeling," Journal of Quantitative Spectroscopy \& Radiative Transfer, vol. 81, no. 1-4, pp. 167-182, 2003.

[16] K. B. Fournier, A. Y. Faenov, T. A. Pikuz et al., "Identification and precise measurements of the wavelengths of high-n transitions in N-, O-, and F-like Zn ions," Journal of Physics $B$, vol. 36, no. 18, pp. 3787-3796, 2003.

[17] A. Y. Faenov, T. A. Pikuz, and I. Y. Skobelev, "X-ray spectroscopic observations of a superdense plasma in nanoparticles irradiated by superintense femtosecond laser radiation," Journal of Experimental and Theoretical Physics Letters, vol. 80, pp. 730-733, 2004.

[18] K. B. Fournier, A. Y. Faenov, T. A. Pikuz et al., "Analysis of high-n dielectronic Rydberg satellites in the spectra of Na-like Zn XX and Mg-like Zn XIX," Physical Review E, vol. 70, no. 1, Article ID 016406, 2004.

[19] M. J. May, P. Beiersdorfer, J. Dunn et al., "Accurate wavelength measurements and modeling of Fe XV to Fe XIX spectra recorded in high-density plasmas between 13.5 and $17 \AA$," Astrophysical Journal, vol. 158, no. 2, pp. 230-241, 2005.

[20] J. Colgan, J. Abdallah, A. Y. Faenov et al., "Model calculations and measurements of the emission of a barium plasma in the spectral range of high-n rydberg levels in a near Ni-like state," Journal of Physics B, vol. 43, no. 17, Article ID 175701, 2010.

[21] L. Reale, A. Lai, A. Tucci et al., "Differences in X-ray absorption due to cadmium treatment in Saponaria Officinalis leaves," Microscopy Research and Technique, vol. 64, no. 1, pp. 21-29, 2004.

[22] T. Pikuz, A. Faenov, I. Skobelev et al., "Easy spectrally tunable highly efficient X-ray backlighting schemes based on spherically bent crystals," Laser and Particle Beams, vol. 22, no. 3, pp. 289-300, 2004.

[23] C. Bellecci, L. Coniglio, M. Francucci et al., "Laser-plasma soft-X-rays analysis by means of a CCD camera: A first application to digital micro-radiography," Nuovo Cimento della Societa Italiana di Fisica B, vol. 121, no. 8, pp. 811-822, 2007.

[24] K. W. D. Ledingham and P. A. Norreys, "Nuclear physics merely using a light source," Contemporary Physics, vol. 40, no. 6, pp. 367-383, 1999.

[25] T. Tajima and J. M. Dawson, "Laser electron accelerator," Physical Review Letters, vol. 43, no. 4, pp. 267-270, 1979.

[26] A. Pukhov, Z. M. Sheng, and J. Meyer-ter-Vehn, "Particle acceleration in relativistic laser channels," Physics of Plasmas, vol. 6, no. 7, pp. 2847-2854, 1999.

[27] M. Gavrila, Advances in Atomic, Molecular and Optical Physics: Atoms in Intense Laser Fields, Academic Press, New York, NY, USA, 1992.

[28] P. M. Celliers, G. W. Collins, L. B. Da Silva et al., "Shockinduced transformation of liquid deuterium into a metallic fluid," Physical Review Letters, vol. 84, no. 24, pp. 5564-5567, 2000.

[29] K. B. Fournier, A. Y. Faenov, T. A. Pikuz et al., "Influence of optical thickness and hot electrons on Rydberg spectra of Nelike and F-like copper ions," Physical Review E, vol. 67, no. 1, Article ID 016402, 2003.

[30] R. W. Falcone and M. M. Murnane, "Proposal for a femtosecond X-ray light source," AIP Conference Proceedings, vol. 147, pp. 81-85, 1986.

[31] M. M. Murnane, H. C. Kapteyn, M. D. Rosen, and R. W. Falcone, "Ultrafast X-ray pulses from laser-produced plasmas," Science, vol. 251, no. 4993, pp. 531-536, 1991.

[32] C. Bellecci, I. Bellucci, P. Gaudio, S. Martellucci, G. Petrocelli, and M. Richetta, "Beam characterization of a high power Nd:YAG-GLASS laser," in Proceedings of the 6th International Workshop on Laser Beam and Optics Characterization (LBOC '6), Munich, Germany, June 2001.

[33] M. Francucci, "Sistemi per la generazione di raggi X da plasma indotto da laser e possibili applicazioni," Ph.D. thesis, University of Rome "Tor Vergata”, 2005.

[34] A. Y. Faenov, S. A. Pikuz, A. I. Erko et al., "High-performance $\mathrm{X}$-ray spectroscopic devices for plasma microsources investigations," Physica Scripta, vol. 50, pp. 333-338, 1994.

[35] I. Y. Skobelev, A. Y. Faenov, and B. A. Bryunetkin, "Investigating the emission properties of plasma structures with $\mathrm{X}$-ray imaging spectroscopy," Journal of Experimental and Theoretical Physics, vol. 81, no. 4, pp. 692-718, 1995.

[36] B. K. F. Young, A. L. Osterheld, D. F. Price et al., "Highresolution X-ray spectrometer based on spherically bent crystals for investigations of femtosecond laser plasmas," Review of Scientific Instruments, vol. 69, no. 12, pp. 4049-4053, 1998.

[37] J. R. Janesick and S. T. Elliot, "Astronomical CCD imaging and reduction," in Astronomical Society of the Pacific Conference Series, S. B. Howell and D. H. McNamara, Eds., vol. 23 of Managing Editors of Conference Series, Bookcrafters, 1992. 
[38] D. Nanni, R. Viotti, M. Badiali, A. M. Di Lellis, and M. Ferrari, "A new $30 \mathrm{~cm}$ three-reflection telescope for wide-field Astronomy on the Antarctic Plateau," in Proceedings of the Future research Direction and Visions for Astronomy, vol. 4835 of Proceedings of SPIE, pp. 192-202, Waikoloa, Hawaii, USA, August 2002.

[39] G. Agnelli, D. Nanni, S. Cola, A. Vignato, A. Di Lellis, and C. D. La Padula, "A thermo electric cooled $2 \mathrm{k} \times 2 \mathrm{k}$ CCD imaging camera for wide-field telescopes," in Proceedings of the Optical Astronomical Instrumentation, vol. 3355 of Proceedings of SPIE, pp. 703-712, Kona, Hawaii, USA, March 1998.

[40] A. Zigler, P. G. Burkhalter, D. J. Nagel et al., "Measurement of energy penetration depth of subpicosecond laser energy into solid density matter," Applied Physics Letters, vol. 59, no. 5, pp. 534-536, 1991.

[41] Z. Jiang, J. C. Kieffer, J. P. Matte et al., "X-ray spectroscopy of hot solid density plasmas produced by subpicosecond high contrast laser pulses at $10^{18}-10^{19} \mathrm{~W} / \mathrm{cm}^{2}$," Physics of Plasmas, vol. 2, no. 5, pp. 1702-1711, 1995.

[42] B. K. F. Young, B. G. Wilson, D. F. Price, and R. E. Stewart, "Measurement of $\mathrm{x}$-ray emission and thermal transport in near-solid-density plasmas heated by 130 fs laser pulses," Physical Review E - Statistical Physics, Plasmas, Fluids, and Related Interdisciplinary Topics, vol. 58, no. 4, pp. 4929-4936, 1998.

[43] K. B. Fournier, B. K. F. Young, S. J. Moon et al., "Characterization of time resolved, buried layer plasmas produced by ultrashort laser pulses," Journal of Quantitative Spectroscopy \& Radiative Transfer, vol. 71, no. 2-6, pp. 339-354, 2001.

[44] H. R. Griem, Spectral Line Broadening by Plasmas, Academic Press, New York, NY, USA, 1974.

[45] R. C. Mancini, A. S. Shlyaptseva, P. Audebert et al., "Stark broadening of satellite lines in silicon plasmas driven by femtosecond laser pulses," Physical Review E - Statistical Physics, Plasmas, Fluids, and Related Interdisciplinary Topics, vol. 54, no. 4, pp. 4147-4154, 1996.

[46] U. Andiel, K. Eidmann, P. Hakel et al., "Demonstration of aluminum K-shell line shifts in isochorically heated targets driven by ultrashort laser pulses," Europhysics Letters, vol. 60, no. 6, pp. 861-867, 2002.

[47] K. Eidmann, U. Andiel, F. Pisani et al., "K-shell spectra from hot dense aluminum layers buried in carbon and heated by ultrashot laser pulses," Journal of Quantitative Spectroscopy \& Radiative Transfer, vol. 81, no. 1-4, pp. 133-146, 2003.

[48] P. Audebert, R. Shepherd, K. B. Fournier et al., "Time-resolved plasma spectroscopy of thin foils heated by a relativisticintensity short-pulse laser," Physical Review E, vol. 66, no. 6, Article ID 066412, 2002.

[49] R. Shepherd, P. Audebert, H. K. Chen et al., "Satellite and opacity effects on resonance line shapes produced from shortpulse laser heated foils," Journal of Quantitative Spectroscopy \& Radiative Transfer, vol. 81, no. 1-4, pp. 431-440, 2003.

[50] Y. Ralchenko et al., 16th APS Topical Conference on Atomic Processes in Plasmas, AIP Conference Proceedings, Melville, NY, USA, 2009.

[51] S. H. Glenzer, K. B. Fournier, B. G. Wilson, R. W. Lee, and L. J. Suter, "Ionization balance in inertial confinement fusion hohlraum plasmas," Journal of Quantitative Spectroscopy \& Radiative Transfer, vol. 71, no. 2-6, pp. 355-363, 2001.

[52] B. Lipschultz, D. G. Whyte, J. Irby, B. Labombard, and G. M. Wright, "Hydrogenic retention with high-Z plasma facing surfaces in Alcator C-Mod," Nuclear Fusion, vol. 49, no. 4, Article ID 045009, 2009.
[53] J. Abdallah, R. E. H. Clark, and R. D. Cowan, "Los Alamos Manual,” No. LA 11436-M-I, Los Alamos National Laboratory, 1988.

[54] R. E. H. Clark, J. Abdallah Jr., and J. B. Mann, "Integral and differential cross sections for electron impact ionization," Astrophysical Journal, vol. 381, no. 2, pp. 597-600, 1991.

[55] J. Abdallah Jr., H. L. Zhang, C. J. Fontes, D. P. Kilcrease, and B. J. Archer, "Model comparisons for high-Z non-LTE steady-state calculations," Journal of Quantitative Spectroscopy \& Radiative Transfer, vol. 71, no. 2-6, pp. 107-116, 2001.

[56] N. H. Magee, J. Abdallah Jr., J. Colgan et al., "Los Alamos opacities: Transition from LEDCOP to ATOMIC," in 14th Topical Conference on Atomic Processes in Plasma, J. S. Cohen, S. Mazevet, and D. P. Kilcrease, Eds., AIP Conference Proceedings, pp. 168-179, New York, NY, USA, 2004.

[57] S. Mazevet and J. Abdallah, "Mixed UTA and detailed line treatment for mid-Z opacity and spectral calculations," Journal of Physics B, vol. 39, no. 16, article no. 022, pp. 34193429, 2006.

[58] B. L. Henke, F. G. Fujiwara, M. A. Tester, C. H. Dittmore, and M. A. Palmer, "Low-energy X-ray response of photographic films. II. Experimental characterization," Journal of the Optical Society of America B, vol. 1, no. 6, pp. 828-849, 1984.

[59] T. Holstein, "Imprisonment of resonance radiation in gases," Physical Review, vol. 72, no. 12, pp. 1212-1233, 1947.

[60] T. Holstein, "Imprisonment of resonance radiation in gases. II," Physical Review, vol. 83, no. 6, pp. 1159-1168, 1951.

[61] J. P. Apruzese, "An analytic Voigt profile escape probability approximation," Journal of Quantitative Spectroscopy \& Radiative Transfer, vol. 34, no. 5, pp. 447-452, 1985.

[62] R. Doron, E. Behar, M. Fraenkel et al., "High-resolution x-ray spectrum of a laser-produced barium plasma in the 9.10-9.36Å wavelength range," Physical Review A - Atomic, Molecular, and Optical Physics, vol. 58, no. 3, pp. 1859-1866, 1998.

[63] R. Doron, M. Fraenkel, P. Mandelbaum, A. Zigler, and J. L. Schwob, "X-ray spectrum emitted by laser-produced barium plasma in the 8 to $13.5 \AA$ wavelength range," Physica Scripta, vol. 58 , no. 1, pp. 19-24, 1998.

[64] V. S. Belyaev, V. I. Vinogradov, A. S. Kurilov et al., "On the role of prepulses during solid target heating by picosecond laser pulses," Journal of Experimental and Theoretical Physics, vol. 96, no. 5, pp. 897-903, 2003.

[65] T. Auguste, P. D'Oliveira, S. Hulin et al., "The role of the prepulse in cluster heating by a high-power femtosecond laser pulse," Journal of Experimental and Theoretical Physics Letters, vol. 72, pp. 38-41, 2000.

[66] A. S. Shlyaptseva, S. B. Hansen, V. L. Kantsyrev et al., "Advanced spectroscopic analysis of 0.8-1.0-MA Mo x pinches and the influence of plasma electron beams on L-shell spectra of Mo ions," Physical Review E, vol. 67, no. 2, Article ID 026409, 2003.

[67] A. Bar-Shalom, M. Klapisch, and J. Oreg, "Hullac, an integrated computer package for atomic processes in plasmas," Journal of Quantitative Spectroscopy \& Radiative Transfer, vol. 71, no. 2-6, pp. 169-188, 2001.

[68] D. Mihalas, Stellar Atmosheres, Freeman, San Francisco, Calif, USA, 1978.

[69] F. B. Rosmej, D. H. H. Hoffmann, M. Geißel et al., "Advanced $\mathrm{x}$-ray diagnostics based on an observation of high-energy Rydberg transitions from autoionizing levels in dense laserproduced plasmas," Physical Review A. Atomic, Molecular, and Optical Physics, vol. 63, no. 6, Article ID 063409, 2001.

[70] G. V. Brown, P. Beiersdorfer, D. A. Liedahl, K. Widmann, and S. M. Kahn, "Laboratory measurements and modeling of the 
Fe XVII X-ray spectrum," Astrophysical Journal, vol. 502, no. 2, pp. 1015-1026, 1998.

[71] G. V. Brown, P. Beiersdorfer, D. A. Liedahl, K. Widmann, S. M. Kahn, and E. J. Clothiaux, "Laboratory measurements and identification of the Fe XVIII-XXIV L-shell X-ray line emission," Astrophysical Journal, vol. 140, no. 2, pp. 589-607, 2002.

[72] L. Reale, A. Lai, I. Bellucci et al., "Microradiography as a tool to detect heavy metal uptake in plants for phytoremediation applications," Microscopy Research and Technique, vol. 69, no. 8, pp. 666-674, 2006. 


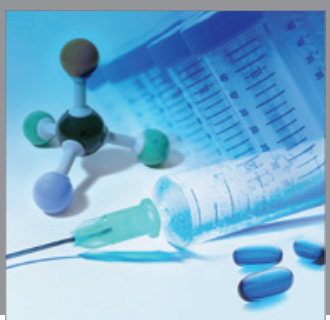

International Journal of

Medicinal Chemistry

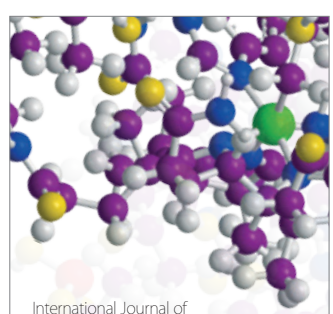

Carbohydrate Chemistry

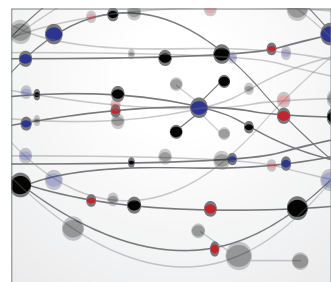

The Scientific World Journal
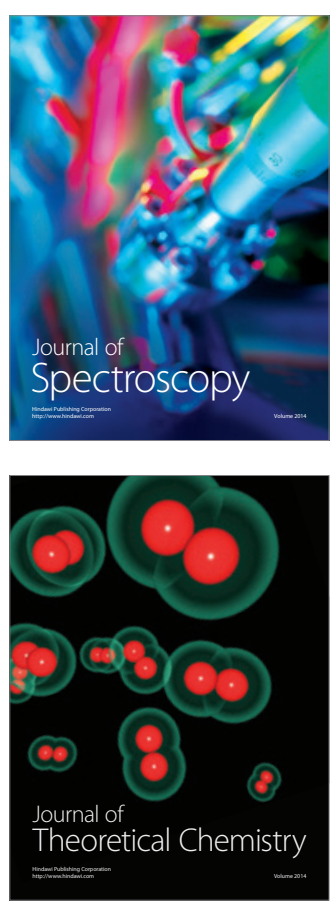
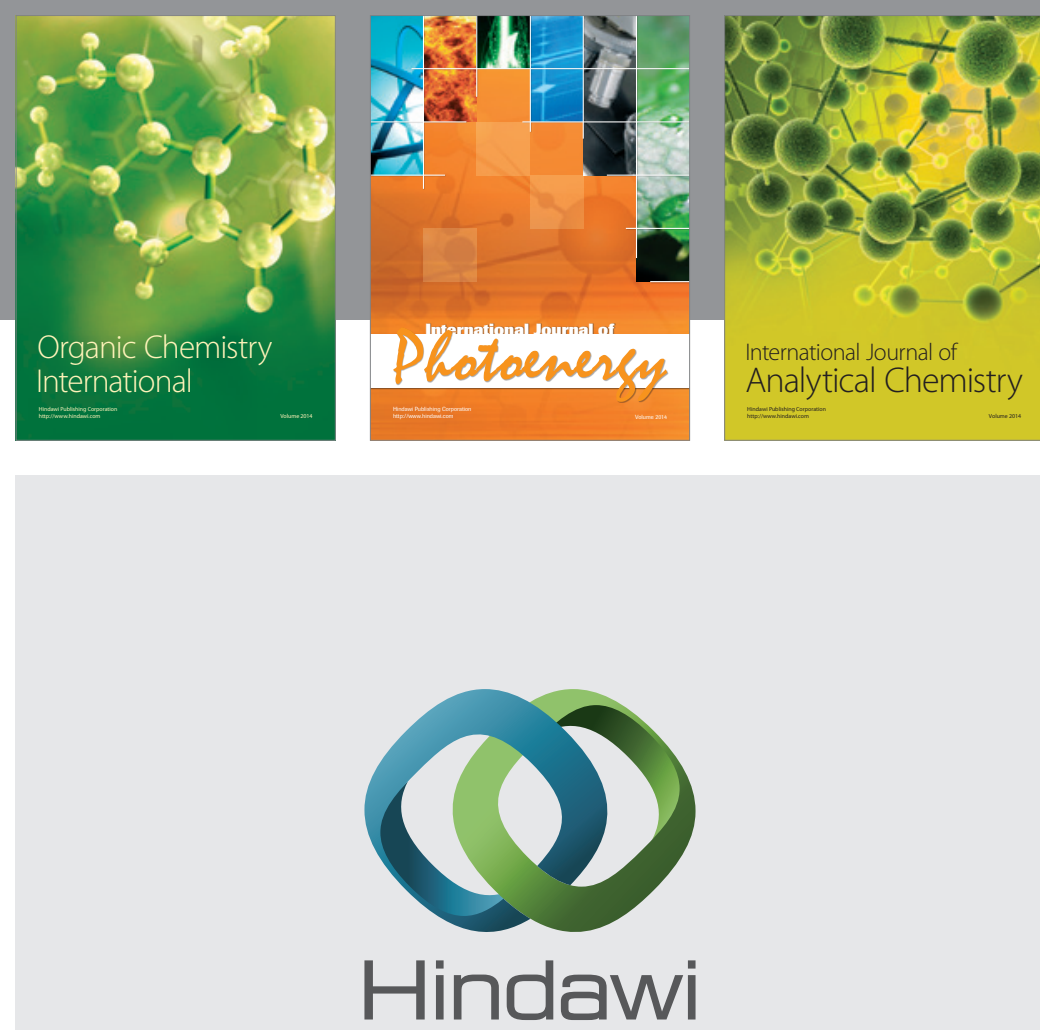

Submit your manuscripts at

http://www.hindawi.com
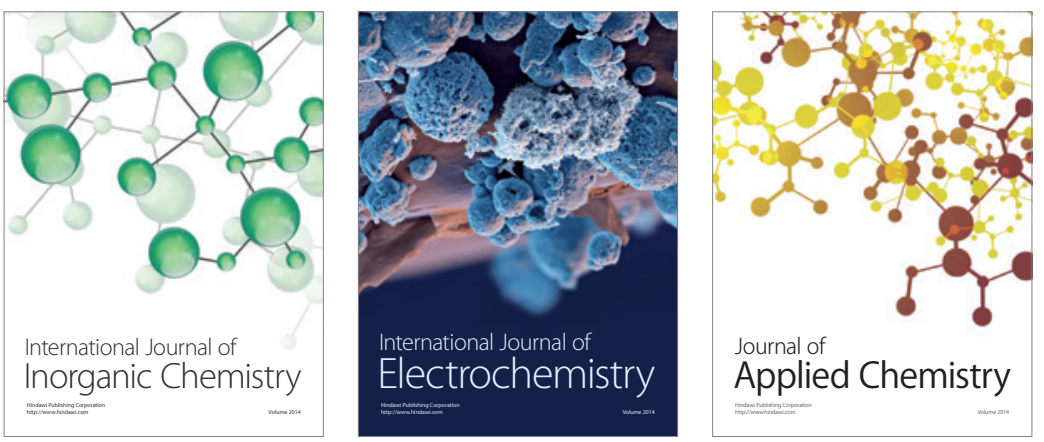

Journal of

Applied Chemistry
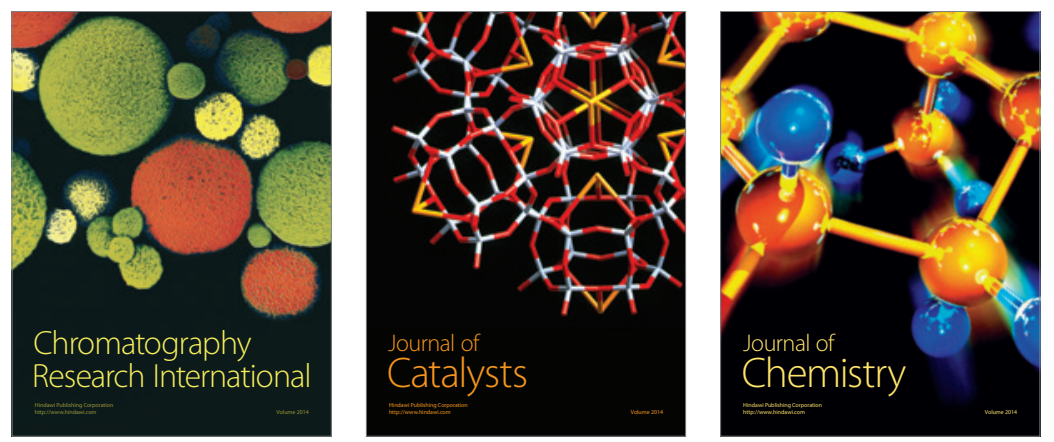
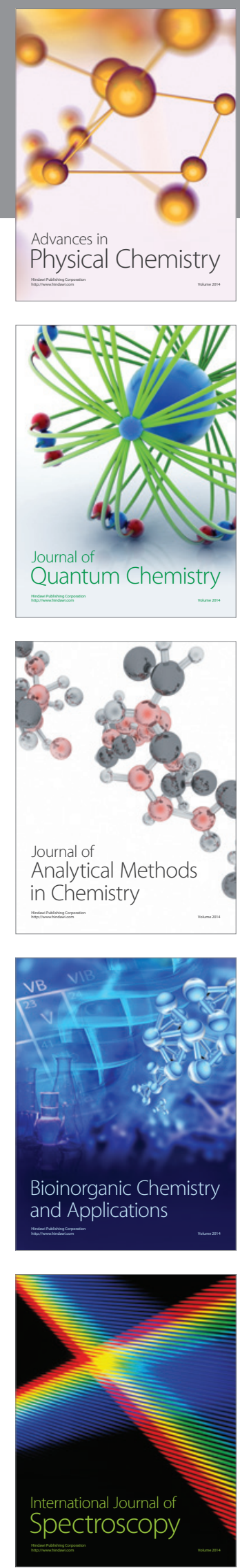